\title{
A comparative Study of Diatom Flora of the Taleghan River in Iran with Other Studies Around the World
}

\author{
Ali Naseri ${ }^{1,2 *}$ and Mostafa Noroozi ${ }^{3}$ \\ ${ }^{1}$ Department of Environment, Alborz Province, Iran \\ ${ }^{2}$ Department of Biology, Science and Research Branch, Islamic Azad University, Iran \\ ${ }^{3}$ Department of Biotechnology, University of Alzahra, Iran \\ Submission: April 27, 2021; Published: June 16, 2021 \\ Corresponding author: Ali Naseri, Department of Biology, Science and Research Branch, Islamic Azad University, Tehran, Iran
}

\begin{abstract}
It is important to compare the flora of different parts of the world which have the various environmental condition. The flora of Taleghan dam and River was identified from November 2017 to October 2018 and the result is compared with 15 regions of recent studies in different continents. The flora of Africa with 66 percent common taxa was the highest similar to our study. Based on the comparison the Ulnaria ulna (Nitzsch) Compère, Navicula cryptotenella Lange-Bertalot Gomphonema acuminatum Ehrenberg and Nitzschia palea (Kützing) W Smith was the cosmopolitan species and Achnanthidium eutrophilum (Lange-Bertalot) Lange-Bertalot, Frustulia tugelae Cholnoky, Nitzschia acula (Kützing) Kützing in Rabenhors, and some other species were rare taxon. 45 out of 203 diagnosed species and genus Seminavis D.G.Mann is new record for diatoms flora of Iran. Also, the species Cymbella pernodensis Maillard, Cymbella novazeelandiana Krammer and Achnanthidium druartii Rimet \& Couté were new records for Asia.
\end{abstract}

Keywords: Diatom; Cosmopolitan; Flora; Sefid Rud; Iran

\section{Introduction}

The changeability of different organisms including the diatoms in various habitats shows the biodiversity comprising species, genetic, and ecosystem diversity. It is necessary to understand the biodiversity to utilize the natural resources for human applications. There is a mutual relation between biodiversity and environment as the alteration in the physicochemical variables, impacts on the diversity of organisms and frequently are responsible for fluctuations the species assemblages. The diatoms are widespread organisms that occurred in various environments like soil, marine, freshwater, and even in extreme conditions. Mann [1] estimated their species number between 30,000 and 200,000 as of the most species-rich in the algal group. They prefer special environmental conditions, such as many other organisms which besides their fast reproduction and their resistant siliceous cell wall (useful for the rebuilding of previous environments) make them a good candidate as bioindicator. Therefore, it is important to study the flora of diatom communities that are influenced by the environmental condition and land-water interactions [2].
It is feasible to evaluate the environment in the aquatic ecosystem with the help of species diversity. Also, the diversity of species is an effective factor in ecosystem functioning, food chain, integrity, and process. The environmental condition such as temperature, humidity, geology, physicochemical elements, substrates (stone, sands, plants, and etc.), anthropogenic factors, water velocity and light intensity influences on the diatom community and their species richness [3,4]. According to the phylogenetic tree the epiphytic and epilithic lifestyle is an ancestral character in which many planktonic diatom species have been evolved from their lineage [5]. The major evolution (lifestyle, morphology, and ecology) of different diatom classes have occurred in the benthic lifestyle. The majority of diatom species live in benthic communities due to their heavy silicified frustule.

Comparing the present flora with the previous studies can reflect climate change and anthropogenic influences on the earth. Iran with 1.6 million $\mathrm{km}^{2}$ has a diverse climate and the Zagros 
Mountains from South part to North and Alborz Mountain from East to West [6]. Outstanding studies on the flora of algae in Iran were performed by $[7,8]$. Compére [9] has mentioned that Rabenhorst [10] has published the list of freshwater diatoms from South Persia. Leonard has collected some samples from Dašt-e Kavir, Dašt-e Lut and Jazmuriyãn in 1972 and Compére [9] has identified 300 algae species and varieties which $99 \%$ of them belonged to Bacillariophyceae [9]. New records of species including Nitzschia curvata Compére, Nitzschia iranica Compére and Navicula egregia Hust. fa. elongata Compére was introduced.

During the five kilometers long sampling area of Taleghan River, 203 Taxa were identified which reflects the rich diatom flora of Iran. Compared to other floristic studies in Iran and other areas (in the same conditions such as: the time of study, sampling substrates, stations number, and etc.), the Taleghan area has the highest diatom diversity. The purpose of the present study was to compare the diatom flora of Taleghan River and Taleghan Dam with 15 other floristic studies in the world.

\section{Material and Methods}

Taleghan River is one of the main tributaries of Sefid Rud, the second-longest river in Iran with approximately $670 \mathrm{~km}$ length. It rises in the Alborz Mountain in the northwest of Iran and flows into the Caspian Sea. Taleghan River begins in the Kahar and Kandovan mountains located in the western part of the Alborz domain. It is one of the longest rivers in the center of Iran with $180 \mathrm{~km}$ length; they form the Shah Roud River after joining to Alamut River. This study was carried out at eight sites on the Taleghan River and Taleghan Dam from November 2017 to October 2018 (Table 1,Figure 1). The number of sediments were $160 \mathrm{ml}$, collected from each station in three replicates. The sediment samples were fixed with a $4 \%$ formaldehyde solution in the sampling field and then transferred to the laboratory. Ten $\mathrm{ml}$ of suspension was prepared from the samples based on the protocol Van der Werff[11].

The samples were treated with $25 \mathrm{ml}$ of hydrogen peroxide $\left(\mathrm{H}_{2} \mathrm{O}_{2}\right)\left(1.5 \mathrm{~h}\right.$ at $\left.100{ }^{\circ} \mathrm{C}\right)$ and then $10 \mathrm{ml}$ hydrochloric acid ( $\left.\mathrm{HCl}\right)(2$ $\mathrm{h}$ at $120^{\circ} \mathrm{C}$ ) to remove organic matters. The oxidized by-products were removed by recurrent and settling with distilled water. To neutralize electrostatic charges on the suspended particles, one drop of $10 \% \mathrm{NH}_{4} \mathrm{CL}$ was added for every $10 \mathrm{~mL}$ of suspension [12]. Coverslips were prepared with diatom suspension and installed on slides in Naphrax glue. The Olympus microscope (Model CX31) via 100x oil immersion was used to investigate the diatom cells. Finally, the Canon camera (Model EOS 5D Mark III) was used to capture the images. The slides were examined to evaluate the abundance of species based on 300 valves counts for each site. Each month, three permanent slides were prepared for eight stations (Table 1, Figure 1).

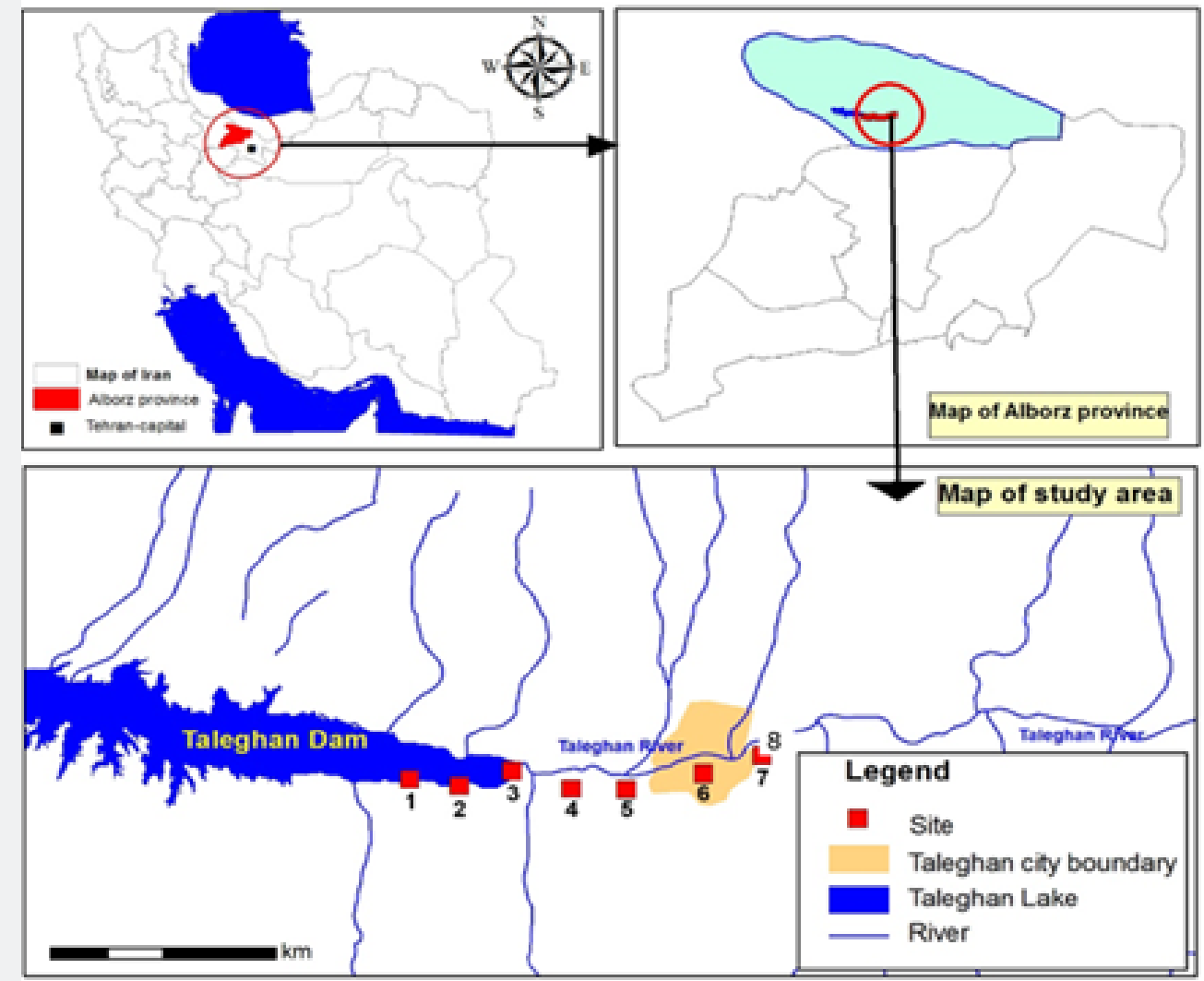

Figure 1: The map of Iran, and locations of sampling sites. 
Table 1: Sampling sites along the Taleghan River.

\begin{tabular}{|c|c|c|}
\hline Site & Elevation $(\mathbf{m})$ & Latitude \& Longitude \\
\hline 1 & 1788 & $36^{\circ} 10.1^{\prime} \mathrm{N}, 50^{\circ} 42.65^{\prime} \mathrm{E}$ \\
\hline 2 & 1791 & $36^{\circ} 10.9^{\prime} \mathrm{N}, 50^{\circ} 42.8^{\prime} \mathrm{E}$ \\
\hline 3 & 1794 & $36^{\circ} 10.1^{\prime} \mathrm{N}, 50^{\circ} 8 \mathrm{E}$ \\
\hline 4 & 1795 & $36^{\circ} 10^{\prime} \mathrm{N}, 50^{\circ} 44.5^{\prime} \mathrm{E}$ \\
\hline 5 & 1797 & $36^{\circ} 10^{\prime} \mathrm{N}, 50^{\circ} 44^{\prime} \mathrm{E}$ \\
\hline 6 & 1817 & $36^{\circ} 10.2^{\prime} \mathrm{N}, 50^{\circ} 46^{\prime} \mathrm{E}$ \\
\hline $7 \& 8$ & 1832 & $36^{\circ} 10.3^{\prime} \mathrm{N}, 50^{\circ} 46.6^{\prime} \mathrm{E}$ \\
\hline
\end{tabular}

\section{Physicochemical Analysis}

To analyze physicochemical parameters, $120 \mathrm{ml}$ of water from each station were collected. The parameters (cations, anions, $\mathrm{SiO}_{2}$, salinity, biological oxygen demand (BOD), chemical oxygen demand (COD), total dissolved solids (TDS), turbidity (nephelometric turbidity unit (NTU), $\mathrm{PO}_{4}^{2-}, \mathrm{NO}_{3}{ }^{2-}$, and $\mathrm{Mg}^{2+}$ ) were examined in the laboratory of the Mabna company (Karaj) according to American Public Health Association [13], and the electrical conductivity (EC), temperature (T), dissolved oxygen (DO) and oxidation-reduction potential (ORP) were measured in situ at station sites [14]. COD was measured by the optical method device, and with Hach Dr 5000. The local temperature, $\mathrm{pH}$ and DO, were measured via the Hach HQ30 analyzer, Nitrate, and phosphate parameters via the Hach Dr 2800 spectrophotometer, and the turbidity was measured by Wagtech turbidity detector.

\section{Results and Discussion}

Table 3 shows the physicochemical parameters of the water of stations. In the Taleghan stations, 203 taxa were identified which belong to 25 families and 49 genera (Table 2). The percentage of each class of diatoms is as below: Bacillariophyceae with $88 \%$, Cosinodiscophyceae with $8 \%$, and Mediophyceae with $4 \%$. The percentage of the diatoms family included Bacillariaceae 21\% (6 genera, 42 species), Navicullaceae 17\% (4 genera, 34 species), Gomphonemataceae 11\% (4 genera, 24 species), Cymbellaceae 10\% (4 genera, 21 species), Achnanthidiaceae 5\% (2 genera, 10 species), each of Catenulaceae, Fragillariaceae, Tabellariaceae, and Surirellaceae had 1, 2,4 genera and 8 to 9 species (each of them $4 \%$ ) respectively, and each of the nineteen other families less than 3\% species accounted for them (Figure 2). Nitzschia $17 \%$ (32 spp.) was the most represented genus, being Navicula, with 26 species (14\%) in the second.

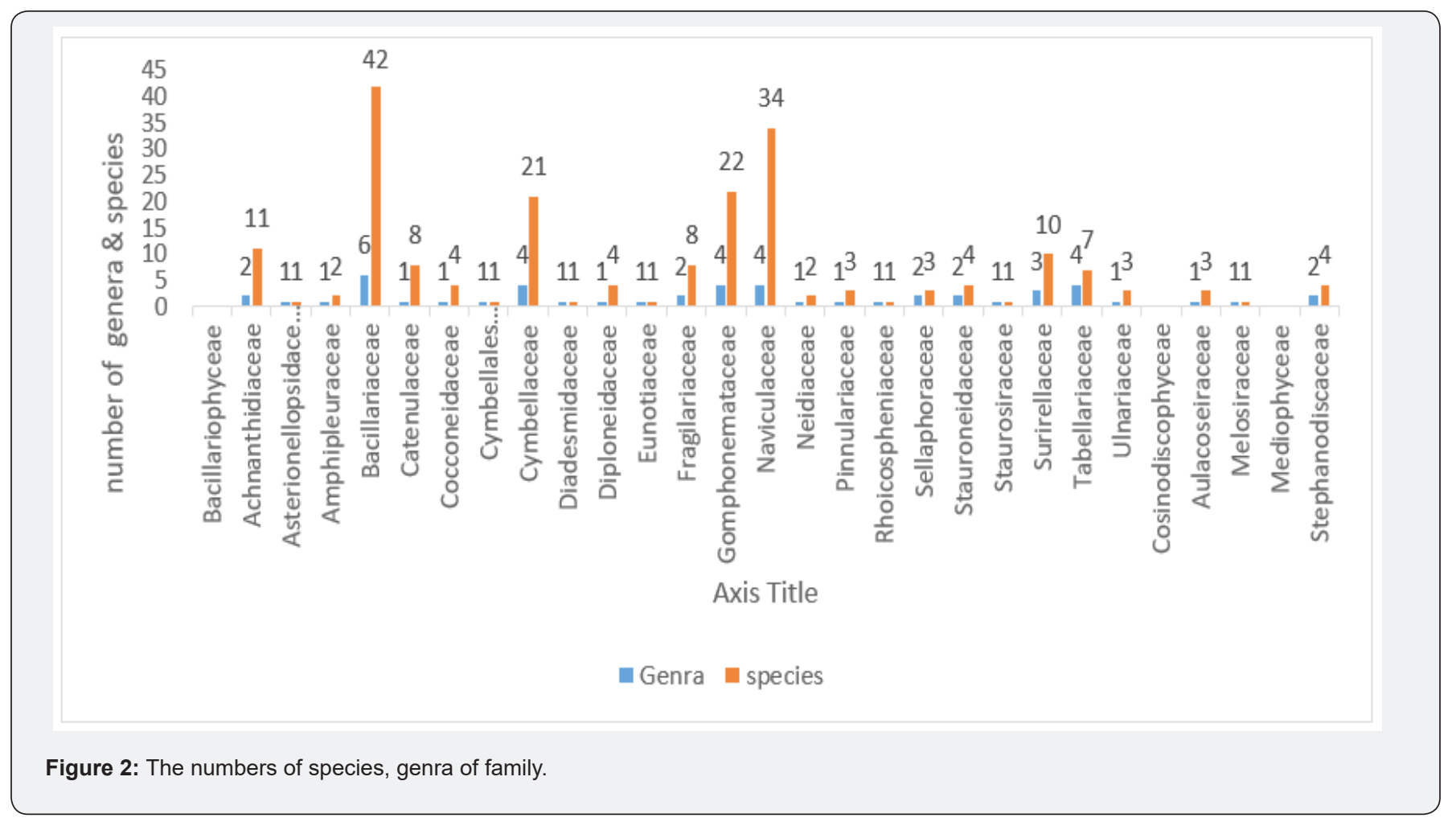


Table 2: Lists and comparison of the taxa of the Taleghan Dam and River in comparison with 15 regions.

\begin{tabular}{|c|c|c|c|c|c|c|c|c|c|c|c|c|c|c|c|}
\hline Taxa Taleghan River (Iran) & $\begin{array}{c}\text { B. } \\
\text { A. } \\
\text { I }\end{array}$ & $\begin{array}{l}\text { K. } \\
\text { R. } \\
\text { I }\end{array}$ & $\begin{array}{l}\text { A. } \\
\text { F. } \\
\text { I. }\end{array}$ & $\begin{array}{l}\text { A. } \\
\text { F. } \\
\text { I } \\
\text { r }\end{array}$ & $\begin{array}{l}\mathbf{S} . \\
\mathbf{R}\end{array}$ & $\begin{array}{c}\text { L. } \\
\text { N. } \\
\text { I }\end{array}$ & $\begin{array}{l}\text { S. } \\
\text { D. } \\
\text { F }\end{array}$ & $\begin{array}{l}\text { V. } \\
\mathbf{E}\end{array}$ & $\begin{array}{l}\text { C. } \\
\text { R. } \\
\text { C }\end{array}$ & $\begin{array}{l}\text { T. } \\
\text { M. } \\
\text { S. } \\
\text { M }\end{array}$ & $\begin{array}{l}\text { E. } \\
\text { C. } \\
\text { R }\end{array}$ & $\begin{array}{l}\text { N. } \\
\text { U. } \\
\text { S }\end{array}$ & $\begin{array}{l}\text { S. } \\
\text { B }\end{array}$ & $\begin{array}{l}\text { P. } \\
\text { B }\end{array}$ & S. \\
\hline \multicolumn{16}{|l|}{ Bacillariophyceae } \\
\hline \multicolumn{16}{|l|}{ Achnanthidiaceae } \\
\hline Achnanthidium affine (Grunow) Czarnecki & & & & & $\mathrm{p}$ & & & & & & & $\mathrm{p}$ & & & $\mathrm{p}$ \\
\hline Achnanthidium crassum (Hustedt) Potapova \& Ponader * & & & & & & & $\mathrm{p}$ & & & & & & & & $\mathrm{p}$ \\
\hline A. druartii Rimet \& Couté** & & & & & & & $\mathrm{p}$ & & & & & & & & \\
\hline A. eutrophilum (Lange-Bertalot) Lange-Bertalot* & & & & & & & $\mathrm{p}$ & & & & & $\mathrm{p}$ & & & $\mathrm{p}$ \\
\hline A. macrocephalum (Hustedt) Round\& Bukhtiyarova* & & & & & & & & & & & & & & & $\mathrm{p}$ \\
\hline A. minutissimum (Kützing) Czarnecki & & $\mathrm{P}$ & & & $\mathrm{p}$ & & & $\mathrm{p}$ & $\mathrm{p}$ & $\mathrm{p}$ & $\mathrm{p}$ & $\mathrm{p}$ & $\mathrm{p}$ & $\mathrm{p}$ & $\mathrm{p}$ \\
\hline $\begin{array}{l}\text { A. saprophilum (H.Kobayashi \& Mayama) Round \& Bukhti- } \\
\text { yarova* }\end{array}$ & & & & & $\mathrm{p}$ & & & & & & & $\mathrm{p}$ & $\mathrm{p}$ & & $\mathrm{p}$ \\
\hline $\begin{array}{l}\text { Achnanthidium standeri (Cholnoky) J.C.Taylor, E.Morales \& } \\
\text { L.Ector }\end{array}$ & & & & & & & & & & & & & & & $\mathrm{P}$ \\
\hline \multicolumn{16}{|l|}{ Achnanthidium sp. } \\
\hline $\begin{array}{l}\text { Planothidium frequentissimum (Lange-Bertalot) Lange- Ber- } \\
\text { talot }\end{array}$ & $\mathrm{P}$ & & & & $\mathrm{P}$ & & & & $\mathrm{P}$ & & & $\mathrm{P}$ & $\mathrm{P}$ & & $\mathrm{P}$ \\
\hline P. rhombiculum Lange-Bertalot* & & & & & & & & & & & $\mathrm{p}$ & & & & \\
\hline \multicolumn{16}{|l|}{ Asterionellopsidaceae } \\
\hline $\begin{array}{l}\text { Psammothidium pseudoswazii (J.R.Carter) L.Bukhtiyarova \& } \\
\text { Round* }\end{array}$ & & & & & & & & & & & & & & & $\mathrm{P}$ \\
\hline \multicolumn{16}{|l|}{ Amphipleuraceae } \\
\hline Frustulia tugelae Cholnoky* & & & & & & & & & & & & & & & $\mathrm{p}$ \\
\hline F. vulgaris (Thwaites) De Toni & & $\mathrm{P}$ & $\mathrm{p}$ & & $\mathrm{p}$ & & & & $\mathrm{p}$ & & $\mathrm{p}$ & $\mathrm{p}$ & & & $\mathrm{p}$ \\
\hline \multicolumn{16}{|l|}{ Bacillariaceae } \\
\hline Bacillaria paxillifera (O.F.Müller) T.Marsson & & & $\mathrm{p}$ & $\mathrm{p}$ & & & & $\mathrm{p}$ & & & & $\mathrm{p}$ & & & $\mathrm{p}$ \\
\hline Denticula kuetzingii Grunow & & $\mathrm{p}$ & & & $\mathrm{p}$ & & & & & & & $\mathrm{p}$ & & & $\mathrm{p}$ \\
\hline D. subtilis Grunow & $\mathrm{p}$ & & & $\mathrm{p}$ & & & & & & & & $\mathrm{p}$ & & & $\mathrm{p}$ \\
\hline Denticula sundayensis Archibald* & & & & & & & & & & & & & & & $\mathrm{p}$ \\
\hline Hantzschia amphioxys (Ehrenberg) Grunow & $\mathrm{P}$ & $\mathrm{p}$ & $\mathrm{p}$ & $\mathrm{p}$ & $\mathrm{p}$ & & & & & & $\mathrm{p}$ & $\mathrm{p}$ & $\mathrm{p}$ & $\mathrm{p}$ & $\mathrm{p}$ \\
\hline \multicolumn{16}{|l|}{ Hantzschia sp. } \\
\hline Mayamaea permitis (Hustedt) Bruner \& Medlin & & & & & & & & & & & $\mathrm{p}$ & $\mathrm{p}$ & & & $\mathrm{p}$ \\
\hline Nitzschia.acicularis (Kützing) W. Smith & & & $\mathrm{p}$ & $\mathrm{p}$ & $\mathrm{p}$ & $\mathrm{P}$ & & & & & $\mathrm{p}$ & $\mathrm{p}$ & & & $\mathrm{p}$ \\
\hline Nitzschia acula (Kützing) Kützing in Rabenhorst* & & & & & & & & & & & & $\mathrm{p}$ & & & \\
\hline N. amphibia Grunow. & $\mathrm{P}$ & $\mathrm{p}$ & $\mathrm{p}$ & & $\mathrm{p}$ & & & & $\mathrm{P}$ & $\mathrm{P}$ & & $\mathrm{p}$ & & & $\mathrm{p}$ \\
\hline N. capitellata Hustedt in Pascher & & & $\mathrm{p}$ & & $\mathrm{p}$ & & & & & & & $\mathrm{p}$ & & & $\mathrm{p}$ \\
\hline N. clausii Hantzsch & & & $\mathrm{p}$ & $\mathrm{p}$ & $\mathrm{p}$ & & & & & $\mathrm{P}$ & & $\mathrm{p}$ & & & $\mathrm{p}$ \\
\hline N. communis Rabenhorst & & & $\mathrm{p}$ & & $\mathrm{p}$ & & & & & & & $\mathrm{p}$ & & & $\mathrm{p}$ \\
\hline N. dissipata (Kützing) Rabenhorst & $\mathrm{P}$ & $\mathrm{p}$ & $\mathrm{p}$ & $\mathrm{p}$ & $\mathrm{p}$ & & & & $\mathrm{P}$ & $\mathrm{P}$ & $\mathrm{p}$ & $\mathrm{p}$ & & & $\mathrm{p}$ \\
\hline N. dissipata var. media (Kützing) Grunow & & $\mathrm{p}$ & & & $\mathrm{p}$ & & & & & & & & & & $\mathrm{p}$ \\
\hline N. draveillensis Coste \& Ricard * & & & & & & & $\mathrm{p}$ & & & & & $\mathrm{p}$ & & & $\mathrm{p}$ \\
\hline N. filiformis (W. Smith) Van Heurck & & & $\mathrm{p}$ & $\mathrm{p}$ & $\mathrm{p}$ & & & $\mathrm{p}$ & & & & $\mathrm{p}$ & & & $\mathrm{p}$ \\
\hline
\end{tabular}


Oceanography \& Fisheries Open access Journal

\begin{tabular}{|c|c|c|c|c|c|c|c|c|c|c|c|c|c|}
\hline N. frustulum (Kützing) Grunow & $\mathrm{p}$ & & $\mathrm{P}$ & $\mathrm{p}$ & $\mathrm{p}$ & & & & & & $\mathrm{p}$ & & $\mathrm{p}$ \\
\hline N. gracilis Hantzsch, & & & $\mathrm{p}$ & $\mathrm{p}$ & $\mathrm{p}$ & & $\mathrm{p}$ & & & & $\mathrm{p}$ & & $\mathrm{p}$ \\
\hline N. heufleriana Grunow & $\mathrm{p}$ & & $\mathrm{P}$ & $\mathrm{p}$ & $\mathrm{p}$ & & & & & & $\mathrm{p}$ & & $\mathrm{p}$ \\
\hline N. iremissa Cholnoky* & & & & & & & & & & & & & $\mathrm{p}$ \\
\hline N. linearis (Agardh) W. Smith & $\mathrm{p}$ & $\mathrm{p}$ & $\mathrm{P}$ & & $\mathrm{p}$ & & & $P$ & $\mathrm{P}$ & $\mathrm{p}$ & $\mathrm{p}$ & & $\mathrm{p}$ \\
\hline N. littorea Grunow* & & & & & & & & & & & $\mathrm{p}$ & & $\mathrm{p}$ \\
\hline N. microcephala Grunow & & & & & $\mathrm{p}$ & & & & $\mathrm{P}$ & & $\mathrm{p}$ & & $\mathrm{p}$ \\
\hline N. media Hantzsch & & $\mathrm{P}$ & & & $\mathrm{P}$ & & & & & & & & $\mathrm{P}$ \\
\hline \multicolumn{14}{|l|}{ Nitzschia oligotraphenta (Lange-Bertalot) Lange-Bertalot* } \\
\hline N. palea (Kützing) W. Smith & $\mathrm{p}$ & & $\mathrm{P}$ & $\mathrm{p}$ & $\mathrm{p}$ & $\mathrm{p}$ & $\mathrm{p}$ & $\mathrm{P}$ & $\mathrm{P}$ & & $\mathrm{p}$ & $\mathrm{p}$ & $\mathrm{p}$ \\
\hline N. pura Hustedt* & & & & & $\mathrm{p}$ & & & & & & $\mathrm{p}$ & & $\mathrm{p}$ \\
\hline N. pusilla Grunow & $\mathrm{p}$ & & $\mathrm{P}$ & $\mathrm{p}$ & $\mathrm{p}$ & & & & & & $\mathrm{p}$ & & $\mathrm{p}$ \\
\hline N Nitzschia recta Hantzsch ex Rabenhorst & & $\mathrm{p}$ & $\mathrm{p}$ & $\mathrm{p}$ & $\mathrm{p}$ & & & $\mathrm{P}$ & & & $\mathrm{p}$ & & $\mathrm{p}$ \\
\hline N. sigma (Kützing) W. Smith & & & $\mathrm{p}$ & $\mathrm{p}$ & $\mathrm{p}$ & & & & & & $\mathrm{p}$ & & $\mathrm{p}$ \\
\hline N. sigmoidea (Nitzsch) W. Smith & $\mathrm{p}$ & & & $\mathrm{p}$ & $\mathrm{p}$ & & & & & & $\mathrm{p}$ & & \\
\hline N. sublinearis Hustedt & & & $\mathrm{p}$ & & & & & & & & & & $\mathrm{p}$ \\
\hline N. subtilis Grunow & & $\mathrm{p}$ & & & & & & & & & & & \\
\hline \multicolumn{14}{|l|}{ N. tenuis W. Smith* } \\
\hline N. tsarenkoi Lange-Bertalot* & & & & & & & & & & & & & $\mathrm{p}$ \\
\hline N. umbonata (Ehrenberg) Lange-Bertalot & & & $\mathrm{p}$ & $\mathrm{p}$ & $\mathrm{p}$ & & & & $\mathrm{P}$ & & $\mathrm{p}$ & & $\mathrm{p}$ \\
\hline N. vermicularis (Kützing) Hantzsch & $\mathrm{P}$ & & $\mathrm{P}$ & $\mathrm{p}$ & $\mathrm{p}$ & & & & & & $\mathrm{p}$ & & \\
\hline Tryblionella apiculata W. Gregory & $\mathrm{p}$ & & & & $\mathrm{p}$ & & & & & & $\mathrm{p}$ & & $\mathrm{p}$ \\
\hline T. angustata W. Smith & & & $\mathrm{P}$ & $\mathrm{P}$ & & & & & & $\mathrm{P}$ & $\mathrm{P}$ & & $\mathrm{P}$ \\
\hline T. calida (Grunow) D.G. Mann & $\mathrm{p}$ & & & & $\mathrm{p}$ & & & & & & $\mathrm{p}$ & & $\mathrm{p}$ \\
\hline T. hungarica (Grunow) Frenguelli & $\mathrm{p}$ & & & & $\mathrm{p}$ & & & & & & $\mathrm{p}$ & & $\mathrm{p}$ \\
\hline \multicolumn{14}{|l|}{ Catenulaceae } \\
\hline Amphora copulata (Kützing) Schoeman \& R.E.M. Archibald & $\mathrm{p}$ & & & $\mathrm{p}$ & & & & & & & $\mathrm{p}$ & & $\mathrm{p}$ \\
\hline A. inariensis Krammer* & & & & & & & & & & & $\mathrm{p}$ & & \\
\hline \multicolumn{14}{|l|}{ A. minutissima W. Smith } \\
\hline A. ovalis (Kützing) Kützing & $\mathrm{p}$ & $\mathrm{p}$ & $\mathrm{P}$ & $\mathrm{p}$ & $\mathrm{p}$ & & $\mathrm{p}$ & & & & $\mathrm{p}$ & & $\mathrm{p}$ \\
\hline A. pediculus (Kützing) Grunow & & $\mathrm{p}$ & $\mathrm{p}$ & $\mathrm{p}$ & $\mathrm{p}$ & & $\mathrm{p}$ & & $\mathrm{p}$ & $\mathrm{p}$ & $\mathrm{p}$ & & $\mathrm{p}$ \\
\hline A. veneta (Kützing) Levkov & & $\mathrm{p}$ & $\mathrm{p}$ & $\mathrm{p}$ & $\mathrm{p}$ & $\mathrm{P}$ & $\mathrm{p}$ & & & & $\mathrm{p}$ & & $\mathrm{p}$ \\
\hline \multicolumn{14}{|l|}{ Amphora sp1 } \\
\hline \multicolumn{14}{|l|}{ Amphora sp2 } \\
\hline \multicolumn{14}{|l|}{ Cocconeidaceae } \\
\hline Cocconeis placentula var. euglypta (Ehrenberg) Grunow & & & $\mathrm{p}$ & $\mathrm{p}$ & $\mathrm{p}$ & & $\mathrm{p}$ & $\mathrm{p}$ & & & $\mathrm{p}$ & & $\mathrm{p}$ \\
\hline C. pediculus Ehrenberg & $\mathrm{p}$ & $\mathrm{p}$ & $\mathrm{p}$ & $\mathrm{p}$ & $\mathrm{p}$ & & & & $\mathrm{p}$ & & $\mathrm{p}$ & & $\mathrm{p}$ \\
\hline C. placentula Ehrenberg & $\mathrm{p}$ & & $\mathrm{p}$ & $\mathrm{p}$ & $\mathrm{p}$ & $\mathrm{p}$ & & & $\mathrm{p}$ & $\mathrm{p}$ & & & $\mathrm{p}$ \\
\hline C. lineata Ehrenberg & & $\mathrm{p}$ & $\mathrm{p}$ & & $\mathrm{p}$ & & $\mathrm{p}$ & & & $\mathrm{p}$ & $\mathrm{p}$ & & $\mathrm{p}$ \\
\hline \multicolumn{14}{|l|}{ Cymbellales incertae sedis (Family) } \\
\hline Gomphonella olivacea (Hornemann) Rabenhorst. & & & $\mathrm{P}$ & & $\mathrm{P}$ & & & & & & & & $\mathrm{P}$ \\
\hline \multicolumn{14}{|l|}{ Cymbellaceae } \\
\hline Cymbella affiniformis Krammer & & $\mathrm{p}$ & & & & & & & & & $\mathrm{p}$ & & \\
\hline
\end{tabular}


Oceanography \& Fisheries Open access Journal

\begin{tabular}{|c|c|c|c|c|c|c|c|c|c|c|c|c|c|c|c|}
\hline C.cf. excisa Kützinge & & $\mathrm{p}$ & & & $\mathrm{p}$ & & & & & & & $\mathrm{p}$ & & & $\mathrm{p}$ \\
\hline C.cf. neocistula Krammer & & $\mathrm{P}$ & & & & & & & & & & $\mathrm{p}$ & & & $\mathrm{p}$ \\
\hline C.cistula (Ehrenberg) 0. Kirchner & $\mathrm{P}$ & & $\mathrm{p}$ & $\mathrm{p}$ & $\mathrm{p}$ & $\mathrm{p}$ & & & & & & & & & $\mathrm{p}$ \\
\hline C.compacta Østrup & & $\mathrm{p}$ & & & & & & & & & & & & & \\
\hline C. diversistigmata Krammer* & & & & & & & & & & & & & & & \\
\hline C. excisiformis Krammer & & & & & & & & & & & & $\mathrm{p}$ & & & \\
\hline C.langebertalotii Krammer & & $\mathrm{P}$ & & & & & $\mathrm{p}$ & & & & & & & & \\
\hline C.novazeelandiana Krammer** & & & & & & & & & & & & & & & \\
\hline C.pernodensis Maillard ** & & & & & & & & & & & & & & & \\
\hline C.stigmaphora Østrup* & & & & & & & & & & & & $\mathrm{p}$ & & & \\
\hline C.subleptoceros Krammer* & & & & & & & & & & & & & & & $\mathrm{p}$ \\
\hline C. turgidula Grun. & & & $\mathrm{p}$ & & & & & & & & & & & & $\mathrm{p}$ \\
\hline Cymbopleura amphicephala (Nägeli ex Kützing) Krammer & & $\mathrm{p}$ & $\mathrm{p}$ & & $\mathrm{p}$ & & & & & & & $\mathrm{p}$ & & & $\mathrm{p}$ \\
\hline C. frequens Schmidt* & & & & & & & & & & & & $\mathrm{p}$ & & & \\
\hline C. langeBertalotii Krammer * & & & & & & & & & & & & & & & \\
\hline C.maggieae Bahls** & & & & & & & & & & & & & & & \\
\hline $\begin{array}{l}\text { Cymbopleura naviculiformis (Auerswald ex Heiberg) Kram- } \\
\text { mer, }\end{array}$ & & & & & & & & & & & & & & & \\
\hline Cymbopleura sp. & & & & & & & & & & & & & & & \\
\hline Didymosphenia geminata (Lyngbye) Mart.Schmidt & & $\mathrm{p}$ & & $\mathrm{p}$ & $\mathrm{p}$ & & & & & & & $\mathrm{p}$ & & & \\
\hline Navicymbula pusilla (Grunow) Krammer* & & & & & & & & & & & & & & & $\mathrm{p}$ \\
\hline Diadesmidaceae & & & & & & & & & & & & & & & \\
\hline Luticola cf. ventricosa (Kützing) D.G. Mann & & & & & & & & & & & & & & & \\
\hline Diploneidaceae & & & & & & & & & & & & & & & \\
\hline Diploneis elliptica (Kützing) Cleve* & & & & $\mathrm{p}$ & $\mathrm{p}$ & & & $\mathrm{p}$ & & $\mathrm{p}$ & & $\mathrm{p}$ & & & $\mathrm{p}$ \\
\hline D. oblongella (Nag.) Cl.Euler & & & $\mathrm{p}$ & $\mathrm{p}$ & & & & $\mathrm{p}$ & & & & $\mathrm{p}$ & & & $\mathrm{p}$ \\
\hline D. smithii (Brebisson) Cleve & & & & $\mathrm{p}$ & & & & & & & & $\mathrm{p}$ & & & $\mathrm{p}$ \\
\hline Diploneis sp. & & & & & & & & & & & & & & & \\
\hline Eunotiaceae & & & & & & & & & & & & & & & \\
\hline Eunotia minor (Kützing.) Grunow & & & $\mathrm{p}$ & & $\mathrm{p}$ & & & & & & $\mathrm{p}$ & $\mathrm{p}$ & & $\mathrm{P}$ & $\mathrm{p}$ \\
\hline Fragilariaceae & & & & & & & & & & & & & & & \\
\hline Fragilaria capucina Desmazières Meso & $\mathrm{p}$ & & $\mathrm{p}$ & $\mathrm{p}$ & $\mathrm{p}$ & & & & $\mathrm{p}$ & $\mathrm{p}$ & & $\mathrm{p}$ & & $\mathrm{P}$ & $\mathrm{p}$ \\
\hline F. capucina var. rumpens (Kutz.) Lange-Bertalot & & & $\mathrm{p}$ & $\mathrm{p}$ & $\mathrm{p}$ & & & & $\mathrm{p}$ & & & & & & $\mathrm{p}$ \\
\hline F. capucina var. rumpens (Kutz.) Lange-Bertalot & & & $\mathrm{p}$ & $\mathrm{p}$ & $\mathrm{p}$ & & & & $\mathrm{p}$ & & & $\mathrm{p}$ & & & $\mathrm{p}$ \\
\hline $\begin{array}{l}\text { Fragilaria tenera var. nanana (Lange-Bertalot) Lange-Ber- } \\
\text { talot \& S. Ulrich }\end{array}$ & & & & $\mathrm{p}$ & $\mathrm{p}$ & & & & & & & & & & $\mathrm{p}$ \\
\hline F. tenera (W. Sm.) Lange-Bertalot & $\mathrm{P}$ & & & & & & & & & & & & & $\mathrm{P}$ & $\mathrm{p}$ \\
\hline Fragilaria sp1. & & & & & & & & & & & & & & & \\
\hline Odontidium hyemale (Roth) Kützing* & & & & & & & & & & & & & & & \\
\hline O. mesodon (Ehrenberg) Kützing & $\mathrm{p}$ & $\mathrm{P}$ & & & & & & & & & & & & & \\
\hline Gomphonemataceae & & & & & & & & & & & & & & & \\
\hline Encyonema caesepitosum Küzing & & $\mathrm{p}$ & $\mathrm{p}$ & $\mathrm{p}$ & $\mathrm{p}$ & & & & & & & & & & $\mathrm{p}$ \\
\hline E. mesianum (Cholnoky) DG Mann & & & & & $\mathrm{p}$ & & & & & & & & & & $\mathrm{p}$ \\
\hline E. minutum (Hilse)D.G. Mann & & $\mathrm{P}$ & & & $\mathrm{p}$ & & & & $\mathrm{p}$ & & $\mathrm{p}$ & $\mathrm{p}$ & & & $\mathrm{p}$ \\
\hline E. silesiacum (Bleisch) D.G. Mann & $\mathrm{p}$ & $\mathrm{P}$ & & & & & & & $\mathrm{p}$ & & & $\mathrm{p}$ & & $\mathrm{P}$ & $\mathrm{p}$ \\
\hline
\end{tabular}




\section{Oceanography \& Fisheries Open access Journal}

\begin{tabular}{|c|c|c|c|c|c|c|c|c|c|c|c|c|c|c|c|}
\hline E. ventricosum (C. Agardh) Grunow & & $\mathrm{P}$ & & & & & & & & & & $\mathrm{p}$ & & & $\mathrm{p}$ \\
\hline \multicolumn{16}{|l|}{ Encyonema sp. } \\
\hline Encyonopsis minuta Krammer \& E. Reichardt & & $\mathrm{P}$ & $\mathrm{p}$ & & & & & & $\mathrm{p}$ & & & $\mathrm{p}$ & & & $\mathrm{p}$ \\
\hline E. subminuta Krammer \& Reichardt & & $\mathrm{P}$ & & & & & & & $\mathrm{p}$ & & & $\mathrm{p}$ & & $\mathrm{P}$ & $\mathrm{p}$ \\
\hline Gomphonema acuminatum Ehrenberg & $\mathrm{p}$ & & $\mathrm{p}$ & $\mathrm{p}$ & $\mathrm{p}$ & $\mathrm{P}$ & & $\mathrm{p}$ & $\mathrm{p}$ & $\mathrm{p}$ & $\mathrm{p}$ & $\mathrm{p}$ & & & $\mathrm{p}$ \\
\hline \multicolumn{16}{|l|}{ G. caperatum Ponader\& Potapova* } \\
\hline G. capitatum Ehrenberg & & $\mathrm{P}$ & & & & & & & $\mathrm{p}$ & & & $\mathrm{p}$ & & & $\mathrm{p}$ \\
\hline G. insigne W.Gregory & & & & & $\mathrm{p}$ & & & & & & & $\mathrm{p}$ & & & \\
\hline \multicolumn{16}{|l|}{ G. louisiananum Kalinsky* } \\
\hline G. minutum (C. Agardh) C. Agardh & & $\mathrm{P}$ & & & $\mathrm{p}$ & & & & $\mathrm{p}$ & & & $\mathrm{p}$ & & & $\mathrm{p}$ \\
\hline G. olivaceoides Hustedt & & & & & $\mathrm{p}$ & & & & & & & $\mathrm{p}$ & & & \\
\hline $\begin{array}{l}\text { G. parvulis (Lange-Bertalot \& E.Reichardt) Lange-Bertalot \& } \\
\text { E.Reichardt* }\end{array}$ & & & & & & & & & & & & $\mathrm{p}$ & & & $\mathrm{p}$ \\
\hline G. parvulum (Kützing) Kützing & $\mathrm{p}$ & & $\mathrm{p}$ & $\mathrm{p}$ & $\mathrm{p}$ & $\mathrm{P}$ & & & $\mathrm{p}$ & $\mathrm{p}$ & $\mathrm{p}$ & $\mathrm{p}$ & & & $\mathrm{p}$ \\
\hline G. pumilum var. rigidum Reichardt \& Lange-Bertalot & & $\mathrm{P}$ & & & $\mathrm{p}$ & & & & $\mathrm{p}$ & & & $\mathrm{p}$ & $\mathrm{p}$ & & $\mathrm{p}$ \\
\hline G. tergestinum (Grunow) Fricke & & $\mathrm{P}$ & $\mathrm{p}$ & $\mathrm{p}$ & $\mathrm{p}$ & & & & & & & & & & \\
\hline \multicolumn{16}{|l|}{ G. venusta passy* } \\
\hline Reimeria sinuata (Gregory) Kociolek \&Stoermer & & $\mathrm{P}$ & & & $\mathrm{p}$ & & & & $\mathrm{P}$ & $\mathrm{p}$ & $\mathrm{p}$ & $\mathrm{p}$ & & & $\mathrm{p}$ \\
\hline R. uniseriata sala Guerrero and Ferrario* & & & & & & & $\mathrm{p}$ & & & & $\mathrm{p}$ & & & & $\mathrm{p}$ \\
\hline \multicolumn{16}{|l|}{ Naviculaceae } \\
\hline Caloneis aequatorialis Hustedt* & & & & & & & & & & & & & & & $\mathrm{p}$ \\
\hline C.bacillum (Grun.) Cleve. & & & $\mathrm{P}$ & $\mathrm{p}$ & $\mathrm{p}$ & & & & $\mathrm{p}$ & & & $\mathrm{p}$ & & & $\mathrm{p}$ \\
\hline C.silicula (Ehrenberg) Cleve. & & $\mathrm{P}$ & $\mathrm{p}$ & $\mathrm{p}$ & $\mathrm{p}$ & & & & & & & $\mathrm{p}$ & & & $\mathrm{p}$ \\
\hline Gyrosigma acuminatum (Kützing) Rabenhorst & $\mathrm{p}$ & & $\mathrm{P}$ & $\mathrm{p}$ & $\mathrm{p}$ & & & & & & & $\mathrm{p}$ & & & $\mathrm{p}$ \\
\hline G. attenuatum (Kutz.) Rabenh. & & & $\mathrm{P}$ & $\mathrm{p}$ & $\mathrm{p}$ & & & & & & & & & & $\mathrm{p}$ \\
\hline G. rautenbachiae Cholnoky* & & & & & & & & & & & & & & & $\mathrm{p}$ \\
\hline G. scalproides (Rabenhorst) Cleve & & & $\mathrm{P}$ & $\mathrm{p}$ & $\mathrm{p}$ & & & & & & & $\mathrm{p}$ & & & $\mathrm{p}$ \\
\hline Navicula amphiceropsis Lange-Bertalot \& U. Rumrich* & & & & & & & & & & & & $\mathrm{p}$ & & & \\
\hline N. antonii Lange-Bertalot & & $\mathrm{P}$ & & & & & & & & & $\mathrm{p}$ & $\mathrm{p}$ & & & $\mathrm{p}$ \\
\hline N. capitatoradiata H. Germain ex Gasse & $\mathrm{p}$ & $\mathrm{P}$ & $\mathrm{p}$ & & $\mathrm{p}$ & & & & $\mathrm{P}$ & $\mathrm{p}$ & & & & & $\mathrm{p}$ \\
\hline N. cincta (Ehrenberg) Ralfs & $\mathrm{p}$ & $\mathrm{P}$ & $\mathrm{p}$ & $\mathrm{p}$ & $\mathrm{p}$ & & & & & & & $\mathrm{p}$ & & & $\mathrm{p}$ \\
\hline N. cryptotenella Lange-Bertalot & & $\mathrm{P}$ & $\mathrm{p}$ & & $\mathrm{p}$ & & & $\mathrm{p}$ & $\mathrm{P}$ & $\mathrm{p}$ & $\mathrm{p}$ & $\mathrm{p}$ & $\mathrm{p}$ & $\mathrm{p}$ & $\mathrm{p}$ \\
\hline N.erifuga Lange-Bertalot & $\mathrm{p}$ & & $\mathrm{p}$ & & & & & & & & & $\mathrm{p}$ & & & $\mathrm{p}$ \\
\hline N. escambia (R.M. Patrick) Metzeltin \& Lange-Bertalot* & & & & & & & & & & & & $\mathrm{p}$ & & & \\
\hline N. germainii J.H. Wallace* & & & & & & & & & & & & $\mathrm{p}$ & & & $\mathrm{p}$ \\
\hline N. gregaria Donkin & $\mathrm{p}$ & & $\mathrm{p}$ & $\mathrm{p}$ & $\mathrm{p}$ & & & & $\mathrm{P}$ & & $\mathrm{p}$ & $\mathrm{p}$ & & & $\mathrm{p}$ \\
\hline \multicolumn{16}{|l|}{ N. notha J.H.Wallace } \\
\hline N. metareichardtiana Lange-Bertalot \& Kusber & $\mathrm{P}$ & $\mathrm{P}$ & & & & & & & & & & $\mathrm{P}$ & & & $\mathrm{P}$ \\
\hline N. novaesiberica Lange-Bertalot* & & & & & & & & & & & & $\mathrm{p}$ & & & \\
\hline \multicolumn{16}{|l|}{ N. pseudolanceolata Lange-Bertalot } \\
\hline N. radiosa Kützing & & & $\mathrm{p}$ & $\mathrm{p}$ & $\mathrm{p}$ & $\mathrm{P}$ & & & & $\mathrm{p}$ & $\mathrm{p}$ & $\mathrm{p}$ & & & $\mathrm{p}$ \\
\hline N. recens (Lange-Bertalot) Lange-Bertalot & $\mathrm{p}$ & & & & $\mathrm{p}$ & & & & & & & $\mathrm{p}$ & & & $\mathrm{p}$ \\
\hline N. rostellata Kützing & & & & & $\mathrm{p}$ & & & & $\mathrm{P}$ & & & $\mathrm{p}$ & & & $\mathrm{p}$ \\
\hline N. simulata Manguin* & & & & & & & & & & & & $\mathrm{p}$ & & & \\
\hline
\end{tabular}


Oceanography \& Fisheries Open access Journal

\begin{tabular}{|c|c|c|c|c|c|c|c|c|c|c|c|c|c|}
\hline N. subrhynchocephala Hustedt* & & & & & & & & & & & & & $\mathrm{p}$ \\
\hline N. tripunctata (O.F. Müller) Bory de SaintVincent & $\mathrm{p}$ & $\mathrm{P}$ & $\mathrm{p}$ & $\mathrm{p}$ & $\mathrm{p}$ & & $\mathrm{p}$ & & $\mathrm{P}$ & $\mathrm{p}$ & $\mathrm{p}$ & & $\mathrm{p}$ \\
\hline N. trivialis Lange-Bertalot & $\mathrm{p}$ & $\mathrm{p}$ & & $\mathrm{p}$ & & & & $\mathrm{P}$ & & & $\mathrm{p}$ & & $\mathrm{p}$ \\
\hline N. vandamii Schoeman\&Archibald* & & & & & & & & & & & $\mathrm{p}$ & & $\mathrm{p}$ \\
\hline N. veneta Kützing & & $\mathrm{p}$ & $\mathrm{p}$ & & $\mathrm{p}$ & & & & & & $\mathrm{p}$ & $\mathrm{p}$ & $\mathrm{p}$ \\
\hline \multicolumn{14}{|l|}{ N. weberi L.L. Bahls } \\
\hline N. zanonii Hust. & & & $\mathrm{p}$ & $\mathrm{p}$ & & & & & & & $\mathrm{p}$ & & $\mathrm{p}$ \\
\hline \multicolumn{14}{|l|}{ Navicula sp 1} \\
\hline \multicolumn{14}{|l|}{ Navicula sp2 } \\
\hline Seminavis strigosa (Hustedt) D. \& E. Amilli* & & & & & & & & & & & & & $\mathrm{p}$ \\
\hline \multicolumn{14}{|l|}{ Neidiaceae } \\
\hline \multicolumn{14}{|l|}{ Neidium sp. } \\
\hline \multicolumn{14}{|l|}{ Pinnulariaceae } \\
\hline Pinnularia acrosphaeria W. Smith & & & $\mathrm{p}$ & $\mathrm{p}$ & $\mathrm{p}$ & & & & & & $\mathrm{p}$ & & $\mathrm{p}$ \\
\hline P. brebissonii (Kützing) Rabenhorst & & & $\mathrm{p}$ & & $\mathrm{p}$ & & & & & $\mathrm{p}$ & & & \\
\hline P. lundii var. linearis Krammer & & & & & & & & & & $\mathrm{p}$ & $\mathrm{p}$ & & \\
\hline \multicolumn{14}{|l|}{ Rhoicospheniaceae } \\
\hline Rhoicosphenia abbreviata (C. Agardh) Lange-Bertalot & $\mathrm{p}$ & $\mathrm{p}$ & $\mathrm{p}$ & & $\mathrm{p}$ & & $\mathrm{p}$ & $\mathrm{P}$ & $\mathrm{p}$ & $\mathrm{p}$ & $\mathrm{p}$ & & $\mathrm{p}$ \\
\hline \multicolumn{14}{|l|}{ Sellaphoraceae } \\
\hline Fallacia pygmaea (Kützing) Stickle \& D.G. Mann & $\mathrm{p}$ & $\mathrm{p}$ & & & $\mathrm{p}$ & & & & & & $\mathrm{p}$ & & $\mathrm{p}$ \\
\hline Sellaphora pupula (Kützing) Mereschkovsky & & $\mathrm{p}$ & & & $\mathrm{p}$ & & & $\mathrm{P}$ & $\mathrm{p}$ & $\mathrm{p}$ & $\mathrm{p}$ & & $\mathrm{p}$ \\
\hline Craticula buderi (Hustedt) DG Mann & & & & & & & & & & & $\mathrm{p}$ & & $\mathrm{p}$ \\
\hline C. buderi (Hustedt) Lange-Bertalot & & & & & & & & & & & $\mathrm{p}$ & & $\mathrm{p}$ \\
\hline C.halophila (Grunow) DG Mann & & & & & & & & & & & $\mathrm{p}$ & & $\mathrm{p}$ \\
\hline Stauroneis smithi Grun. & & & $\mathrm{p}$ & & $\mathrm{p}$ & & & & & $\mathrm{p}$ & $\mathrm{p}$ & & $\mathrm{p}$ \\
\hline \multicolumn{14}{|l|}{ Staurosiraceae } \\
\hline Pseudostaurosira parasitica (W. Smith) Morales & & $\mathrm{p}$ & $\mathrm{p}$ & & & & & & & & & & \\
\hline \multicolumn{14}{|l|}{ Surirellaceae } \\
\hline \multicolumn{14}{|l|}{ Campylodiscus sp. } \\
\hline Surirella angusta Kützing & $\mathrm{p}$ & $\mathrm{p}$ & $\mathrm{p}$ & & $\mathrm{p}$ & & & $\mathrm{P}$ & $\mathrm{p}$ & $\mathrm{p}$ & & & $\mathrm{p}$ \\
\hline S. atomus Turpin* & & & & & & & & & & & $\mathrm{p}$ & & \\
\hline \multicolumn{14}{|l|}{ S. lacrimula J.D. English in English\& Potapova* } \\
\hline S. librile (Ehrenberg) Ehrenberg & $\mathrm{p}$ & & & $\mathrm{p}$ & $\mathrm{p}$ & $\mathrm{P}$ & & & & $\mathrm{p}$ & $\mathrm{p}$ & & $\mathrm{p}$ \\
\hline S. minuta Brébisson ex Kützing & $\mathrm{p}$ & $\mathrm{p}$ & $\mathrm{p}$ & & $\mathrm{p}$ & & & & & $\mathrm{p}$ & $\mathrm{p}$ & & \\
\hline S. ovalis Brébissons Eutroph & $\mathrm{p}$ & & $\mathrm{p}$ & $\mathrm{p}$ & $\mathrm{p}$ & $\mathrm{p}$ & & & & $\mathrm{p}$ & $\mathrm{p}$ & & $\mathrm{p}$ \\
\hline S. tenera W. Gregory & & & $\mathrm{p}$ & $\mathrm{p}$ & & & & & & & $\mathrm{p}$ & & \\
\hline \multicolumn{14}{|l|}{ Surirella sp. } \\
\hline \multicolumn{14}{|l|}{ Tabellariaceae } \\
\hline Asterionella formosa Hassall & & $\mathrm{p}$ & $\mathrm{p}$ & & $\mathrm{p}$ & & & & & $\mathrm{p}$ & & & $\mathrm{p}$ \\
\hline Diatoma moniliformis (Kützing) D.M. Williams & $\mathrm{p}$ & $\mathrm{p}$ & & & $\mathrm{p}$ & & & & & & $\mathrm{p}$ & & \\
\hline D. tenuis C. Agardh & $\mathrm{p}$ & & $\mathrm{p}$ & $\mathrm{p}$ & $\mathrm{p}$ & & & & & & $\mathrm{p}$ & & \\
\hline
\end{tabular}




\section{Oceanography \& Fisheries Open access Journal}

\begin{tabular}{|c|c|c|c|c|c|c|c|c|c|c|c|c|c|c|}
\hline D. vulgaris Bory. & $\mathrm{p}$ & $P$ & $\mathrm{p}$ & $\mathrm{p}$ & & $P$ & & & & & $\mathrm{p}$ & & & $\mathrm{p}$ \\
\hline D. vulgaris var. breve Grun & $\mathrm{p}$ & $\mathrm{P}$ & $\mathrm{p}$ & $\mathrm{p}$ & $\mathrm{p}$ & & & & & & & & & $\mathrm{p}$ \\
\hline Meridion constrictum Ralfs * & & $\mathrm{p}$ & & & $\mathrm{p}$ & & & & & $\mathrm{p}$ & $\mathrm{p}$ & & & \\
\hline Oxyneis binalis (Ehrenberg) Round & $\mathrm{p}$ & & & & & & & & & & & & & \\
\hline \multicolumn{15}{|l|}{ Ulnariaceae } \\
\hline Hannaea arcus (Ehrenberg) Patrick & $\mathrm{p}$ & $\mathrm{P}$ & & $\mathrm{p}$ & $\mathrm{p}$ & & & & & $\mathrm{p}$ & $\mathrm{p}$ & & & \\
\hline Ulnaria biceps (Kützing)P. Compère & & $\mathrm{p}$ & $\mathrm{p}$ & $\mathrm{p}$ & $\mathrm{p}$ & & & & & & $\mathrm{p}$ & & & $\mathrm{p}$ \\
\hline U. ulna (Nitzsch) Compère & $\mathrm{P}$ & $\mathrm{p}$ & $\mathrm{p}$ & $\mathrm{p}$ & & $\mathrm{P}$ & $\mathrm{p}$ & $\mathrm{P}$ & $\mathrm{p}$ & & $\mathrm{p}$ & $\mathrm{p}$ & $\mathrm{P}$ & $\mathrm{p}$ \\
\hline \multicolumn{15}{|l|}{ Cosinodiscophyceae } \\
\hline \multicolumn{15}{|l|}{ Aulacoseiraceae } \\
\hline Aulacoseira granulata (Ehrenberg) Simonsen & & & $\mathrm{p}$ & & & & & & $\mathrm{p}$ & & $\mathrm{p}$ & $\mathrm{p}$ & & $\mathrm{p}$ \\
\hline \multicolumn{15}{|l|}{ Aulacoseira sp1. } \\
\hline \multicolumn{15}{|l|}{ Aulacoseira sp2. } \\
\hline \multicolumn{15}{|l|}{ Melosiraceae } \\
\hline Melosira varians C. Agardh & $\mathrm{p}$ & $\mathrm{P}$ & $\mathrm{p}$ & $\mathrm{p}$ & & & & $\mathrm{p}$ & $\mathrm{p}$ & $\mathrm{p}$ & $\mathrm{p}$ & & & $\mathrm{p}$ \\
\hline \multicolumn{15}{|l|}{ Mediophyceae } \\
\hline \multicolumn{15}{|l|}{ Stephanodiscaceae } \\
\hline Cyclotella atomus Hustedt* & & & & & & & & & & & $\mathrm{p}$ & & & $\mathrm{p}$ \\
\hline Cyclotella meneghiniana Kützing & $\mathrm{p}$ & & $\mathrm{p}$ & & & $\mathrm{p}$ & $\mathrm{p}$ & & $\mathrm{p}$ & & $\mathrm{p}$ & $\mathrm{P}$ & $\mathrm{p}$ & $\mathrm{p}$ \\
\hline Pantocsekiella ocellata (Pantocsek) K.T. Kiss \& Ács & & $\mathrm{p}$ & $\mathrm{p}$ & & & & $\mathrm{p}$ & & & & $\mathrm{p}$ & & & $\mathrm{p}$ \\
\hline Pantocsekiella rossi (H. Håkansson) K.T. Kiss \& E. Ács & & $\mathrm{p}$ & & & & & & & & & & & & \\
\hline
\end{tabular}

Notes: P stand for presence, B.A.I for Balikhli River Northwest Iran; K.R.I: Karaj River in Iran, A.F.I: Algal flora Iran; N.L.I: Neure Lake Iran; A.f.Ir: Algal flora Iraq; S.R: Southern of the Russian; S.d.f: Serbian diatom flora; V.E: Valenciana España; C.R.C: Cali River Colombia; T.M.S.M: tropical mountain streams of Mexico; E.C. R: Elbsandsteingebjrge Czech Republic; N.U. S: Northwestern United States; S.B: southeastern Brazil; P.B: Paraná state Brazil,\& S.A: South Africa. The new records for the flora of Iran are marked by an asterisk and new records to the flora of Asia by two asterisks.

The percentage of other genera are mentioned as: Each of Cymbella and Gomphonema, had 13 species (each of them 7\%), each of Amphora, Surirella, and Achnanthidium with 8 species (4\%), each of the Fragilaria, Cymbopleura, Encyonema, and Diatoma, had 5 to 6 species (3\%), Tryblionella, Caloneis, Denticula, Craticula, Aulacoseira, Gyrosigma, Cocconeis, and Pinnularia, had 3 to 4 species (2\%), 29 other genera had 1 to 2 species (1\%) of the identified taxa (Figure 2). Forty-five out of 203 diagnosed species are new records for the diatom flora of Iran which are marked with an asterisk in Table 2. Also, the genus Seminavis D.G., is new record for Iran. As well, three species were recognized as the new report for Asia including Cymbella novazeelandiana Krammer: $(71,166$; pl.47; pl.51) [15]. Cymbella pernodensis Maillard ex Lange-Bertalot \& Moser [16]: (79; pl. 20; pl.25), and Achnanthidium druartii Rimet \& Couté [17]: (47572: 188, pls. I-III (Figures 3a-f). Due to their low frequency (R: $<1.5 \%)$ in the samples, we cannot provide any better pictures. Cymbella novazeelandiana Kramme (Figure 3a\&b), has only been reported in New Zealand and Australia [18]. It is also a new record for the diatom flora of Asia (Figure 2).

In this study some research of diatom flora was chosen which were representative of continents (Asia, Europe, Africa, America). The study of Brazil diatoms was conducted to increase the biodiversity and ecology of tropical diatoms. In the Czech Republic study, the region includes pristine habitat with clean and unpolluted waters. The sampling in the Serbian study was done from different substrates including epiphyte and epilithic which two genera (Microfissurata and Fistulifera) were new for their flora. The sampling stations in Iraq and its geographical position were highly diverse from cold to hot and mountains to deserts areas which make it special. The characteristics of the sampling area in the United States were diverse regarding $\mathrm{pH}$, temperature, salinity, nutrient, and a vast area which can be the reason for the diversity of diatom species.

Based on the comparison study the Cymbella pernodensis Maillard, has not been reported in Iran [19-30]. It is a freshwater taxon which occurred only in stations $5 \& 6$, in all months except spring with the following environmental conditions: alkaline $\mathrm{pH}$ (8.71), moderate to high conductivity (502- $834 \mu \mathrm{s.cm}{ }^{-1}$ ), oligotrophic to eutrophic conditions, temperature (4.9 - 19.9 $\left.{ }^{\circ} \mathrm{C}\right)$, TDS (164 -335 mg. $\left.\mathrm{l}^{-1}\right)$, BOD (<8 mg. $\left.\mathrm{l}^{-1}\right)$, DO $\left(<10 \mathrm{mg} \cdot \mathrm{l}^{-1}\right)$, ORP

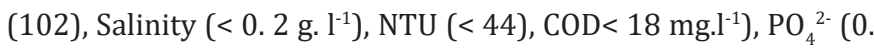
$3-4$ mg.l $\left.{ }^{-1}\right), \mathrm{NO}_{3}{ }^{2-}\left(<3\right.$ mg.l $\left.\mathrm{l}^{-1}\right), \mathrm{Si}\left(2.9-5.1{\mathrm{mg} . \mathrm{l}^{-1}}^{-1}\right), \mathrm{Mg}^{2+}(4.8-16.3$ mg..$^{-1}$ ) (Table 3 ). 


\section{Oceanography \& Fisheries Open access Journal}

Table 3: Seasonal mean and standard deviation of physicochemical parameters from sampling stations.

\begin{tabular}{|c|c|c|c|c|c|}
\hline Parameters & Station & Spring & Autumn & Summer & Winter \\
\hline \multirow{8}{*}{ *TDS $\left(\mathrm{mg} / \mathrm{l}^{-1}\right)$} & 1 & $190 \pm 26.9072$ & $187.4 \pm 7.73821$ & $157 \pm 7.9372$ & $232.34 \pm 59.8776$ \\
\hline & 2 & $204 \pm 19.1572$ & $270.6667 \pm 21.7865$ & $229 . .33 \pm 91.5277$ & $243.67 \pm 55.0121$ \\
\hline & 3 & $174 . .34 \pm 21.4553$ & $269.5333 \pm 24.662$ & $220 \pm 75.7825$ & $328.33 \pm 33.8575$ \\
\hline & 4 & $176.67 \pm 22.8108$ & $317 \pm 9.5393$ & $212 \pm 73.3212$ & $335.33 \pm 40.2036$ \\
\hline & 5 & $117.34 \pm 18.8237$ & $276 \pm 22.33830$ & $212 \pm 69.3974$ & $244.33 \pm 70.5147$ \\
\hline & 6 & $185.67 \pm 25.4230$ & $323.34 \pm 10.6926$ & $211 \pm 73.7360$ & $334.67 \pm 27.2274$ \\
\hline & 7 & $150 \pm 17.0880$ & $301 \pm 44.9777$ & $205.33 \pm 73.3303$ & $355 \pm 55.4616$ \\
\hline & 8 & $190.67 \pm 24.1729$ & $320.6 \pm 15.14375$ & $218.33 \pm 56.3589$ & $382.66 \pm 57.9511$ \\
\hline \multirow{11}{*}{${ }^{*} \mathrm{DO}\left(\mathrm{mg} / \mathrm{l}^{-1}\right)$} & 1 & $9.22 \pm 0.3576$ & $6.367 \pm 0.0723$ & $8.83 \pm 0.5843$ & $8.56 \pm 0.2410$ \\
\hline & 2 & $8.8 \pm 1.1857$ & $7.24 \pm 0.0655$ & $7.56 \pm 0.2650$ & $8.63 \pm 0.900$ \\
\hline & 3 & $9.67 \pm 0.4974$ & $9.87 \pm 0.5351$ & $8.29 \pm 0.7011$ & $9.96 \pm 0.4454$ \\
\hline & 4 & $9.2334 \pm 0.1457$ & $9.12 \pm 1.0484$ & $8.543 \pm 0.444$ & $9.83 \pm 0.6357$ \\
\hline & 5 & $8.79 \pm 0.4214$ & $9.17 \pm 0.8155$ & $8.043 \pm 0.6230$ & $9.73 \pm 0.3450$ \\
\hline & 6 & $8.9 \pm 0.1058$ & $9.37 \pm 1.3150$ & $8.01 \pm 0.5708$ & $9.54 \pm 0.3260$ \\
\hline & 7 & $8.67 \pm 0.3066$ & $8.81 \pm 0.9103$ & $7.98 \pm 0.4119$ & $9.33 \pm 0.1563$ \\
\hline & 8 & $7.64 \pm 0.9544$ & $9.65 \pm 0.4948$ & $6.56 \pm 0.4636$ & $8.23 \pm 0.5838$ \\
\hline & 1 & $8.3 \pm 0.1011$ & $8.3 \pm 0.1890$ & $8.4 \pm 0.1365$ & $8.4 \pm 0.3774$ \\
\hline & 2 & $7.9 \pm 0.1921$ & $7.5 \pm 0.1059$ & $8.4 \pm 0.0754$ & $8.1 \pm 0.0500$ \\
\hline & 3 & $8.2 \pm 0.0346$ & $8.4 \pm 0.0608$ & $8.4 \pm 0.2007$ & $8.5 \pm 0.1193$ \\
\hline \multirow[t]{8}{*}{$\mathrm{pH}$} & 4 & $8.2 \pm 0.0665$ & $8.4 \pm 0.0953$ & $8.5 \pm 0.3827$ & $9.5 \pm 0.94$ \\
\hline & 5 & $8.75 \pm 0.8231$ & $8.6 \pm 0.1550$ & $8.5 \pm 0.1975$ & $8.6 \pm 0.0263$ \\
\hline & 6 & $8.2 \pm 0.0321$ & $8.4 \pm 0.028$ & $8.3 \pm 0.1379$ & $8.5 \pm 0.0251$ \\
\hline & 7 & $8.2 \pm 0.2757$ & $8.0 \pm 0.5257$ & $8.4 \pm 0.3659$ & $8.4 \pm 0.1357$ \\
\hline & 8 & $7.64 \pm 0.4063$ & $7.6 \pm 0.3957$ & $7.9 \pm 0.3280$ & $7.8 \pm 0.1248$ \\
\hline & 1 & $474.33 \pm 68.090$ & $380 \pm 70.5053$ & $412 \pm 20.7846$ & $578 \pm 151.0860$ \\
\hline & 2 & $509 \pm 47.947$ & $448.66 \pm 54.0030$ & $597.66 \pm 152.454$ & $616 \pm 129.5723$ \\
\hline & 3 & $435.33 \pm 54.500$ & $558 \pm 227.3477$ & $544.66 \pm 131.062$ & $820.33 \pm 85.2310$ \\
\hline \multirow[t]{7}{*}{${ }^{*} \mathrm{EC}\left(\mu \mathrm{s} . \mathrm{cm}^{-1}\right)$} & 4 & $441.33 \pm 56.888$ & $610 \pm 170.6135$ & $608.33 \pm 106.7817$ & $836 \pm 100.5385$ \\
\hline & 5 & $291.66 \pm 46.285$ & $565.66 \pm 91.0842$ & $553 \pm 218.2750$ & $609.33 \pm 175.819$ \\
\hline & 6 & $461 \pm 64.280$ & $619.66 \pm 185.9740$ & $620.66 \pm 162.6571$ & $833.33 \pm 67.869$ \\
\hline & 7 & $476 \pm 61.991$ & $627.33 \pm 179.3804$ & $595 \pm .01952$ & $785 \pm 155.9647$ \\
\hline & 8 & $810 \pm 153.948$ & $627 \pm 449.5631$ & $840.33 \pm 95.5527$ & $995.33 \pm 91.664$ \\
\hline & 1 & $13.26 \pm 3.3501$ & $17.16 \pm 5.2519$ & $24.1 \pm 1.1532$ & $6.77 \pm 2.7024$ \\
\hline & 2 & $12.43 \pm 4.5544$ & $14.83 \pm 3.9803$ & $19.57 \pm 2.1361$ & $7.8 \pm 1.3856$ \\
\hline \multirow[t]{10}{*}{$\mathrm{T}(\stackrel{\circ}{ } \mathrm{C})$} & 3 & $9.46 \pm 3.0599$ & $12.8 \pm 7.9680$ & $18.03 \pm 1.0503$ & $4.53 \pm 1.5695$ \\
\hline & 4 & $10.86 \pm 3.4063$ & $13.3 \pm 8.2054$ & $17.5 \pm 0.50$ & $5.33 \pm 1.3316$ \\
\hline & 5 & $11.26 \pm 2.6388$ & $12.3 \pm 7.0760$ & $18.57 \pm 0.9814$ & $5.87 \pm 0.9073$ \\
\hline & 6 & $11.56 \pm 2.8867$ & $11.97 \pm 6.6108$ & $19 \pm 0.7937$ & $5.8 \pm 0.5291$ \\
\hline & 7 & $11.53 \pm 2.1939$ & $11.97 \pm 6.1451$ & $17.4 \pm 2.2052$ & $6.43 \pm 0.4163$ \\
\hline & 8 & $13.26 \pm 3.2254$ & $13.8 \pm 3.9038$ & $16.67 \pm 1.0263$ & $11.2 \pm 1.1135$ \\
\hline & 1 & $81.9 \pm 1.1$ & $84.2 \pm 9.9141$ & $92.17 \pm 10.5396$ & $75.67 \pm 6.8244$ \\
\hline & 2 & $63.07 \pm 14.9887$ & $52.53 \pm 29.0993$ & $75.9 \pm 18.1033$ & $64.67 \pm 6.9873$ \\
\hline & 3 & $78.5 \pm 2.3579$ & $89.83 \pm 3.1973$ & $82.73 \pm 4.1004$ & $88.33 \pm 13.4767$ \\
\hline & 4 & $78.43 \pm 2.6857$ & $87.53 \pm 2.9194$ & $83.67 \pm 4.9328$ & $87.3 \pm 11.9954$ \\
\hline
\end{tabular}


Oceanography \& Fisheries Open access Journal

\begin{tabular}{|c|c|c|c|c|c|}
\hline \multirow[t]{6}{*}{${ }^{*} \mathrm{ORP}$} & 5 & $81.63 \pm 4.1052$ & $92.43 \pm 2.9737$ & $89.8 \pm 5.0318$ & $93.2 \pm 11.5723$ \\
\hline & 6 & $78.9 \pm 2.4248$ & $85.67 \pm 3.2393$ & $87.83 \pm 6.7884$ & $87.17 \pm 15.4997$ \\
\hline & 7 & $76.13 \pm 5.7933$ & $84.33 \pm 2.0305$ & $84.37 \pm 7.2665$ & $82 \pm 6.7201$ \\
\hline & 8 & $49.1 \pm 12.7106$ & $58.47 \pm 1.3316$ & $50.83 \pm 4.4814$ & $45.93 \pm 14.3060$ \\
\hline & 1 & $24.8 \pm 21.9063$ & $217.7863 \pm 0.133$ & $12.11 \pm 4.2353$ & $172.44 \pm 172.485$ \\
\hline & 2 & $160.17 \pm 135.991$ & $249.4 \pm 5.5912$ & $103.77 \pm 131.848$ & $87.82 \pm 105.262$ \\
\hline \multirow[t]{9}{*}{ *NTU } & 3 & $208.83 \pm 228.211$ & $7.873 \pm 5.4337$ & $58.77 \pm 91.9923$ & $100.57 \pm 88.7924$ \\
\hline & 4 & $207.23 \pm 194.208$ & $7.28 \pm 4.1371$ & $65.29 \pm 98.4728$ & $74.83 \pm 101.8168$ \\
\hline & 5 & $64.37 \pm 29.6473$ & $5.27 \pm 6.3918$ & $36.65 \pm 43.6044$ & $28.05 \pm 23.8778$ \\
\hline & 6 & $199.53 \pm 178.643$ & $7.873 \pm 1.5305$ & $48.98 \pm 70.1596$ & $124.12 \pm 183.469$ \\
\hline & 7 & $194.07 \pm 204.784$ & $2.15 \pm 4.0501$ & $42.2 \pm 59.5838$ & $112.17 \pm 180.115$ \\
\hline & 8 & $45.67 \pm 29.67$ & $5.63 \pm 3.1923$ & $41.72 \pm 68.6556$ & $10.63 \pm 15.1046$ \\
\hline & 1 & $0.23 \pm 0.1527$ & $1.65 \pm 2.1250$ & $0.27 \pm 0.0577$ & $0.67 \pm 1.0653$ \\
\hline & 2 & $0.45 \pm 0.3400$ & $2.62 \pm 3.8804$ & $0.38 \pm 0.2804$ & $0.35 \pm 0.4763$ \\
\hline & 3 & $0.42 \pm 0.5054$ & $1.41 \pm 2.1564$ & $0.22 \pm 0.0529$ & $0.73 \pm 0.5507$ \\
\hline \multirow[t]{8}{*}{$\mathrm{PO}_{4}{ }^{2-}$} & 4 & $0.62 \pm 0.5647$ & $1.28 \pm 1.4907$ & $0.26 \pm 0.01$ & $0.40 \pm 0.5169$ \\
\hline & 5 & $0.63 \pm 0.4250$ & $2.25 \pm 1.5173$ & $0.37 \pm 0.0208$ & $0.97 \pm 0.2886$ \\
\hline & 6 & $0.18 \pm 0.1106$ & $1.63 \pm 2.4866$ & $0.31 \pm 0.0854$ & $0.63 \pm 0.3900$ \\
\hline & 7 & $0.16 \pm 0.060$ & $2.16 \pm 3.1525$ & $0.48 \pm 0.2818$ & $0.47 \pm 0.5485$ \\
\hline & 8 & $0.15 \pm 0.0873$ & $2.17 \pm 3.2347$ & $0.31 \pm 0.0230$ & $0.41 \pm 0.5169$ \\
\hline & 1 & $2.91 \pm 1.4705$ & $2.2 \pm 0.7810$ & $1.76 \pm 0.4927$ & $1.4 \pm 0.6000$ \\
\hline & 2 & $3.9 \pm 1.5099$ & $3.7 \pm 2.8583$ & $2.43 \pm 0.4932$ & $1.83 \pm 0.5507$ \\
\hline & 3 & $3.7 \pm 1.1532$ & $3.05 \pm 0.6500$ & $3.02 \pm 0.6353$ & $2.4 \pm 1.1357$ \\
\hline \multirow[t]{7}{*}{$\mathrm{NO}_{3}^{2-}$} & 4 & $3.09 \pm 0.6407$ & $2.6 \pm 0.1732$ & $3.1 \pm 0.3605$ & $2.06 \pm 1.0016$ \\
\hline & 5 & $3.27 \pm 0.3511$ & $2.27 \pm 0.3055$ & $2.85 \pm 1.2020$ & $2.16 \pm 0.7094$ \\
\hline & 6 & $3.47 \pm 0.3511$ & $1.6 \pm 1.0392$ & $2.22 \pm 0.5688$ & $2.93 \pm 0.9018$ \\
\hline & 7 & $3.4 \pm 0.1732$ & $2 \pm 0.8185$ & $2.16 \pm 0.4613$ & $2.8 \pm 1.3$ \\
\hline & 8 & $4.65 \pm 1.0819$ & $2.12 \pm 0.6932$ & $2.55 \pm 1.8183$ & $3.03 \pm 0.4725$ \\
\hline & 1 & $2.71 \pm 0.4517$ & $2.067 \pm 0.327$ & $2.07 \pm 0.3271$ & $3.05 \pm 0.8760$ \\
\hline & 2 & $3.28 \pm 1.1001$ & $4.83 \pm 1.0272$ & $4.83 \pm 1.0272$ & $3.42 \pm 0.8145$ \\
\hline \multirow[t]{9}{*}{$\mathrm{Si}\left(\mathrm{mg} . \mathrm{l}^{-1}\right)$} & 3 & $4.12 \pm 0.7441$ & $4.91 \pm 0.5884$ & $4.91 \pm 0.5884$ & $5.34 \pm 2.4282$ \\
\hline & 4 & $3.87 \pm 0.54500$ & $4.88 \pm 0.5129$ & $4.88 \pm 0.5129$ & $5.45 \pm 2.3696$ \\
\hline & 5 & $3.88 \pm 0.2893$ & $5 \pm 0.4689$ & $5 \pm 0.4689$ & $5.51 \pm 2.6681$ \\
\hline & 6 & $3.85 \pm 0.5023$ & $4.87 \pm 0.5089$ & $4.88 \pm 0.5089$ & $5.54 \pm 2.2360$ \\
\hline & 7 & $3.70 \pm 0.466$ & $5.10 \pm 0.6942$ & $5.10 \pm 0.6942$ & $5.77 \pm 2.9021$ \\
\hline & 8 & $4.15 \pm 0.2753$ & $5.22 \pm 0.7448$ & $5.22 \pm 0.7448$ & $5.85 \pm 2.7319$ \\
\hline & 1 & $14.88 \pm 7.467$ & $13.16 \pm 0.5919$ & $10.85 \pm 2.7484$ & $10.21 \pm 3.0571$ \\
\hline & 2 & $9.92 \pm 2.4159$ & $23.09 \pm 0.8013$ & $9.54 \pm 1.6185$ & $16 \pm 2.9328$ \\
\hline & 3 & $13.12 \pm 3.6345$ & $14.57 \pm 1.6667$ & $9.81 \pm 1.2932$ & $17.6 \pm 2.2170$ \\
\hline \multirow[t]{5}{*}{$\mathrm{Mg}^{2+}\left(\mathrm{mg} / \mathrm{l}^{-1}\right)$} & 4 & $7.36 \pm 3.8797$ & $13.75 \pm 3.1005$ & $8.33 \pm 2.4179$ & $18.88 \pm 9.8057$ \\
\hline & 5 & $7.04 \pm 2.4159$ & $12.96 \pm 0.8313$ & $8.86 \pm 0.7034$ & $12.8 \pm 4.8318$ \\
\hline & 6 & $7.68 \pm 1.92$ & $15.34 \pm 0.9657$ & $6.38 \pm 2.0082$ & $13.76 \pm 5.2872$ \\
\hline & 7 & $7.91 \pm 1.0450$ & $13.7 \pm 1.7776$ & $8.43 \pm 1.7953$ & $14.37 \pm 2.4946$ \\
\hline & 8 & $13.44 \pm 3.4613$ & $18.71 \pm 0.4801$ & $14.38 \pm 3.9607$ & $16.32 \pm 1.92$ \\
\hline
\end{tabular}

*TDS: Total dissolved solids; DO: Disolved oxygen; EC: Electrical conductivity, ORP: Oxidation reduction potential; NTU: Nephelometric turbidity unit 
Achnanthidium druartii Rimet \& Couté [17], have been reported from France and Spain Germany, Netherlands, Serbia: Jovac stream and Đetinja River, and Bulgaria [17,31-34]. This species is considered aggressive and has been invading the rivers of Europe [17]. It has been reported in North America only from rivers in Kentucky and Virginia [35]. This epilithic taxon usually lives on stones, however. Unlike the Rimet's results in the present study it occurred in sediment, so this taxon can be considered as epipelic and epilithic taxon [17]. This taxon has special characters such as a longer valve, lower striae density, and lanceolate shape with subrostrated apices which make it distinguishable from other species like A. convergens Kobayasi, A. deflexum (Reimer) Kingston, and A. japonicum Kobayasi.

Frustulia tugelae Cholnoky, Ref identify [28], (plate. 44), L: 20$26 \mu \mathrm{m}, \mathrm{W}: 4.5-5 \mu \mathrm{m}$, it has dimensions: Valve length 17-26 $\mu \mathrm{m}$, Valve breadth $4-5 \mu \mathrm{m}$, and Striae density $>40.10 \mu \mathrm{m}$ (i.e., not visible in
LM). The type species (holotype) is Frustulia saxonica Rabenhorst and no synonym is currently included in Algae Base. This taxon is a freshwater species (www.algaebase.org) or associated with weakly acidic waters [28]. It has a restrict ecological range, only reported from the eastern mountainous regions of South Africa, and Tasmania $[28,36]$.

A very small diatom linear shape and other individual characteristics with strongly concave valve margins, poorly elongated, rounded to sub-capitate apices and if not focused on, it may not be observed by diatomists in microscopic investigation. In our samples, it occurred in slightly alkaline $\mathrm{pH}$ (7.25), moderate electrolyte to high electrolyte content with conductivity (309- 890 $\mu \mathrm{cm}^{-1}$ ) and eutrophic conditions, with DO (7. 25 -9.74 mg. $\mathrm{l}^{-1}$ ),

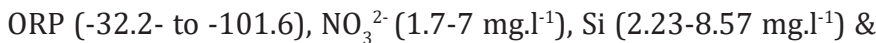
$\mathrm{Mg}^{2+}$ (11.48-24 mg. $\left.{ }^{-1}\right)$. It was observed only in winter and autumn (temperature $4.3-19.7^{\circ} \mathrm{C}$ ) (Figure 3, Table 2).

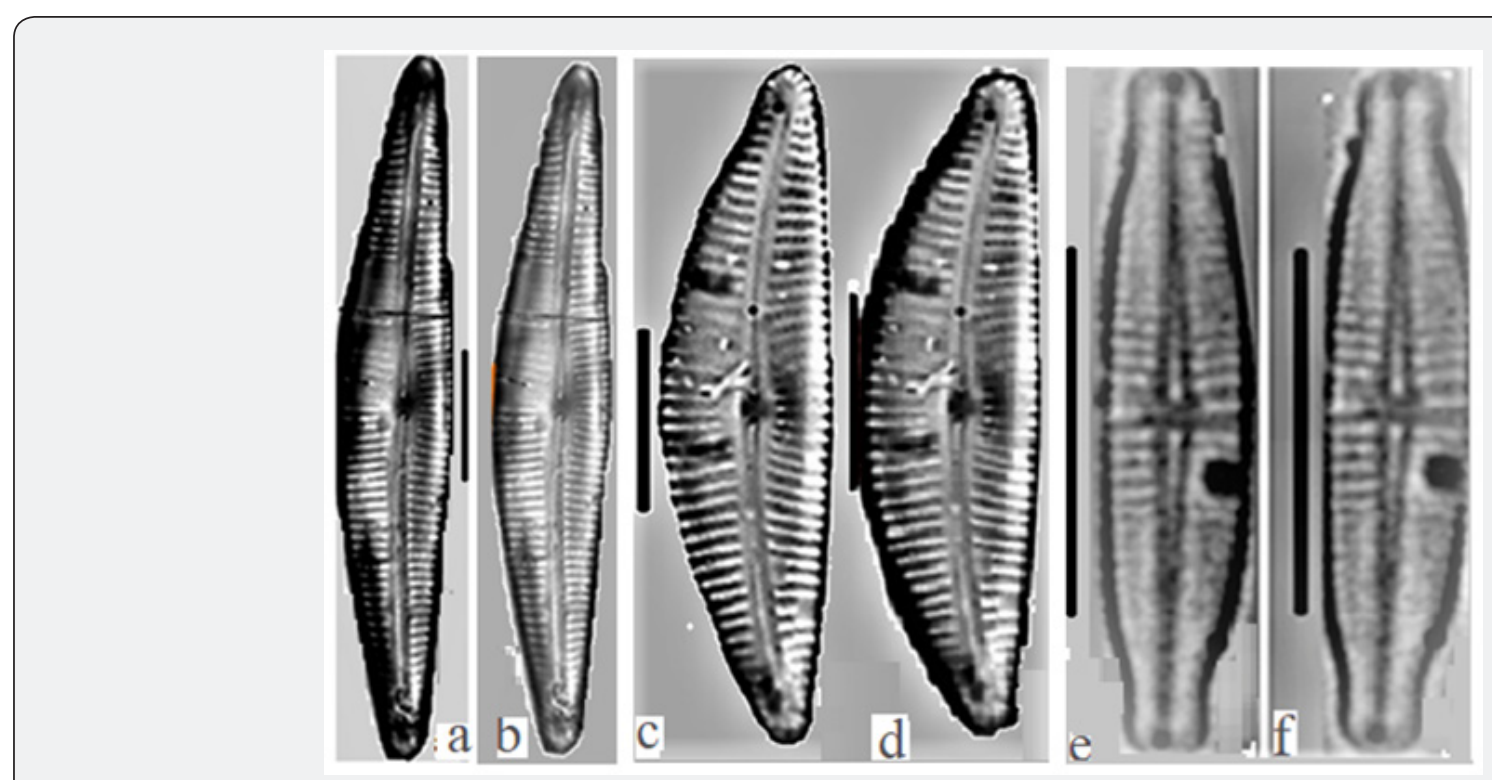

Figure 3: a \& b Cymbella novazeelandiana; c \& d Cymbella pernodensis and e \& f Achnathidium druartii are new records for diatoms flora

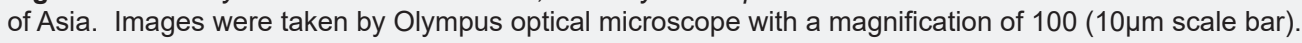

The Seminavis strigosa (Hustedt) Danieledis \& EconomouAmilli in Danielidis \& Mann: it has been reported in New Zealand, North America: NW USA, German, Tasmania, Netherlands, Middle East (Egypt), South-west Asia (India), Spain, North America (Florida), Mexico, and Africa (Ivory Coast) [18,23,31,32,36-39,4143]. The Ulnaria ulna (Nitzsch) Compère is the most frequent species which has been occurred in 12 out of 15 compared studies. Following this species in frequency is Navicula cryptotenella Lange Bertalot, Gomphonema acuminatum Ehrenberg, and Nitzschia palea (Kützing) W Smith, which were common in 11 studies. So, it can be concluded that these species are cosmopolitan and probably they can be seen in the normal environment of whole the world.
Some taxa were only reported from Iran's rivers, but not present in other compared regions, such as Nitzschia subtilis (Kützing) Grunow, N. oligotraphenta Lange Bertalot, A. minutissima W. Smith. Cymbella compacta Østrup, C. diversistigmata Krammer, Gomphonema venusta Passy, Navicula notha Wallace. So, they may be, found in special geographical regions. The other objective was to compare the flora of Taleghan River with other studies in Iran and the other 15 areas in the world (Table 2). The highest and the lowest similarity was between Africa's rivers (66\%) and Serbia's diatoms (3\%) respectively.

Medlin \& Kaczmarska and Medlin have classified the Bacillarophyta to three classes Coscinodiscophyceae, Mediophyceae, and Bacillariophyceae which it is supported by 
the molecular analysis, however, we have used the Guiry \& Guiry diatoms classification [44-46] (Figure 4, Table 3). Probably the high diversity of the Taleghan River can be related to the presence of 10 mines in the upstream which release the inorganic elements.
The assembly of ecosystems and species diversity is affected by anthropogenic activities such as destroying habitats, organic, and inorganic pollution.

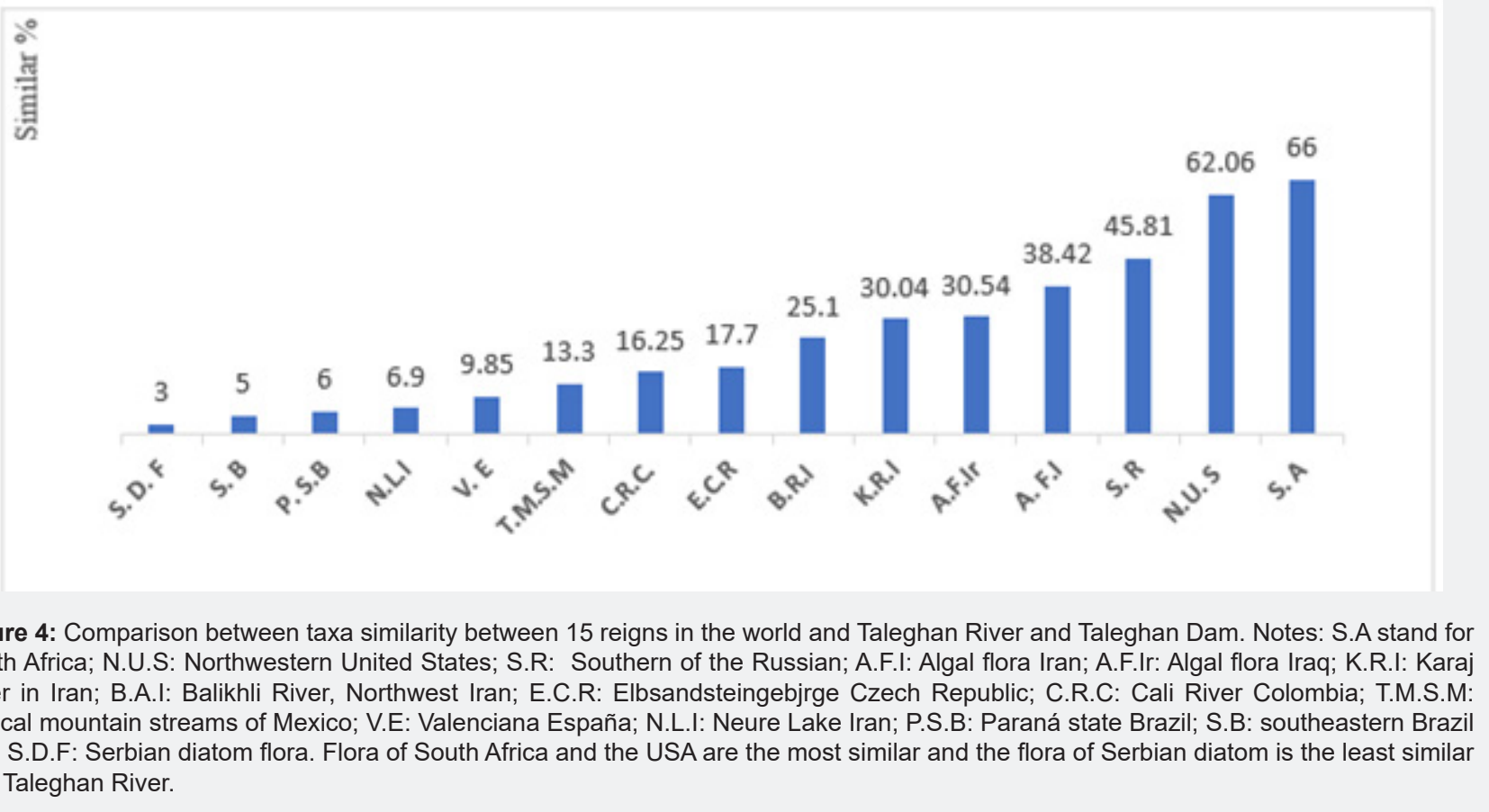

\section{Conclusion}

Based on the performed comparison, the Ulnaria ulna, Navicula cryptotenella, Gomphonema acuminatum and Nitzschia palea was the cosmopolitan species and Achnanthidium standeri, Frustulia tugelae, Nitzschia acula, and some other species were rare taxon. Sixty-four species and two genera were new records for diatoms flora of Iran and for Asia the species Cymbella pernodensis, Cymbella novazeelandiana, and Achnanthidium druartii were new recorded. According to the comparison of Taleghan diatom flora with 15 other environments, it can be concluded that the flora of Africa and the USA are the most similar and the flora of Serbia is the least similar to the Taleghan River. Also, the rare and cosmopolitan species was visible in Table 2.

\section{References}

1. Mann DG (1999) The species concept in diatoms. Phycologia 38(6): 437-495.

2. Flotemersch JE, Stribling JB, Paul MJ (2006) Concepts and Approaches for the Bioassessment of Non-Wadeable Streams and Rivers. United States Environmental Protection Agency, USA.

3. Michelutti N, Holtham AJ, Douglas MSV, Smol JP (2003) Periphytic diatom assemblages from ultra-oligotrophic and UV transparent lakes and ponds on Victoria Island and comparisons with other diatom surveys in the Canadian Arctic. Journal of Phycology 39(3): 465-480.
4. Bouchard G, Gaejwski K, Hamilton PB (2004) Freshwater diatom biogeography in the Canadian Arctic Archipelago. Journal of Biogeography 31(12): 1955-1973.

5. Koistra WHC F, De Stefano M, Mann DG, Medlin K (2006) The Phylogeny of the diatoms. Plant genome: biodiversity and evolution. In: AK Sharma, A Sharma (Eds.) Science Publisher, Enfield, New Hampshire.

6. Akhani H, Mahdavi P, Noroozi J, Zarrinpour V (2013) Vegetation Patterns of the Irano-Turanian Steppe along a 3,000 m Altitudinal Gradient in the Alborz Mountains of Northern Iran. Folia Geobotanica 48: 229-255.

7. Löffler H (1959) Beitrage zur Kenntnis Der Iranischen Binnengewässer I Der Niriz-See und Sein Einzugsgebiet. Internationale Revue der Gesamten Hydrobiologie Und Hydrogr 44(1-4): 227-276.

8. Löffler H (1961) Beitrage zur Kenntnis der Iranischen Binnengewasser. II. Regional-Limnologische Studie Mit Besonderer Berucksichtigung Der Crustaceenfauna. Internationale Revue der gesamten Hydrobiologie und Hydrographie 46(3): 309-406.

9. Compére P (1981) Algues des deserts d Iran. Bulletin Du Jardin Botanique National de Belgique/Bulletin van de National Plantentuin van België 51(1/2): 3-40.

10. Rabenhorst L (1853) Die Süsswasser-Diatomaceen. Leipzig 72.

11. Van der Werff A (1953) A new method of concentrating and cleaning diatoms and other organisms. SIL Proceedings 12(1): 276-277.

12. Mcbride TP (1988) Preparing random distribution of diatom valves on microscope slides. Limnology and Oceanography 33(6): 1627-1629. 
13. APHA (1995) American Public Health Association - Standard methods for the examination of water and wastewater. In: A P H Association (Ed.) (19th edn) Washington.

14. Clesceri LS, Greenberg AE, Trussell RR (1990) Standard Methods for the Examination of Water and Wastewater. In: American Public Health Association, Washington, DC.

15. Krammer K (2002) Diatoms of the European Inland waters and comparable habitats. In: H Lange-Bertalot (ed.), A R A Gantner Verlagll, Ruggell, Pp. 584.

16. Moser G, Steindorf A, Lange-Bertalot H (1995) Neukaledonien Diatomeenflora einer Tropeninsel. Revision der Collection Maillard und Untersuchungen neuen Materials. Bibliotheca Diatomologica 32: 1-340.

17. Rimet F, Couté A, Piuz A, Berthon V, Druart JC (2010) Achnanthidium druartii sp. nov. (Achnanthales, Bacillariophyta), A new species invading European rivers. Vie et Milieu 60: 185-195.

18. Harper M A, Cassie Cooper V, Chang FH, Nelson WA, Broady PA (2012) Phylum ochrophyta: brown and golden-brown algae, diatoms, silicoflagellates, and kin. New Zealand Inventory of Biodiversity. Kingdoms Bacteria, Protozoa, Chromista, Plantae, Fungi. Canterbury University Press, Christchurch 3: 114-163.

19. Panahy-Mirzahasanlou J, Nejadsattari T, Ramezanpour Z, Namin JI, Asri Y (2018) The epilithic and epipelic diatom flora of the Balikhli River, Northwest Iran. Turkish Journal of Botany 42: 518-532.

20. Zarei-Darki B (2009) Taxonomic structure of the algal flora of Iran. Bangladesh Journal of Plant Taxonomy 16(2): 185-194.

21. Nejadsattari T (2005) The diatom flora of lake neure, Iran. Diatom Research 20(2): 313-333.

22. Maulood BK, Hassan FM, Al-Lami AAZ, Toma JJ, Ismail AM (2013) Checklist of algal flora in Iraq.

23. Bahls LL (2009) A Checklist of Diatoms from Inland Waters of the Northwestern United States. Proceedings of the Academy of Natural Sciences of Philadelphia 158: 1-35.

24. Medvedeva LA, Nikulina TV (2014) Catalogue of freshwater algae of the southern part of the Russian Far East. Catalogue of freshwater algae of the Southern part of the Russian Far East 271.

25. Kulikovskiy MS, Glushchenko A, Genkal SI, Kuznetsova I (2016) Identification Book of diatoms from Russia.

26. Costa LF, Wengrat S, Bicudo DC (2017) Diatoms from distinct habitats of a highly heterogeneous reservoir, Billings Complex, southeastern Brazil. Hoehnea 44(4): 559-579.

27. Cantoral-Uriza EA, Sanjurjo MA (2008) Diatomeas (Bacillariophyceae) del marjal Oliva-Pego (Comunidad Valenciana, España). Anales Del Jardin Botanico de Madrid 65: 111-128.

28. Taylor JC, Harding WR, Archibald CGM (2007) An illustrated guide to some common diatom species from South Africa. Report to the Water Research Commission. Pretoria, South Africa 225.

29. Veselá J, Johansen JR (2009) The diatom flora of ephemeral headwater streams in the elbsandsteingebirge region of the Czech Republic. Diatom Research 24(2): 443-477.
30. Heinrich CG, Palacios-Peñaranda ML, Peña-Salamanca E, Schuch M Lobo EA (2019) Epilithic diatom flora in Cali River hydrographical basin, Colombia. Rodriguésia 70: 1-28.

31. Hofmann G, Lange-Bertalot H, Werum M, Klee R (2018) Rote liste und gesamtartenliste der limnischen kieselalgen (Bacillariophyta) Deutschlands. In: Rote liste gefährdeter tiere, Pflanzen und Pilze deutschlands. (Metzing, D). Münster: land wirtschaftsverlag.

32. Veen A, Hof CHJ, Kouwets FAC, Berkhout T (2015) Taxa water management the Netherlands. Laboratory for Hydrobiological Analysis, Rijkswaterstaat.

33. Vidaković DP, Jakovljević OS, Predojević DD, Radovanović S M, SubakovSimić G V, et al. (2018) An updated list of Serbian diatom flora: New recorded taxa. Archives of Biological Sciences 70: 259-275.

34. Ivanov PN (2018) Two new diatom species from family Achnanthidiaceae in Bulgaria: Achnanthidium druartii, an invasive species in Europe and Achnanthidium straubianum, new to Bulgarian diatom flora. Phytologia Balcanica 24(2): 195-199.

35. Potapova MG, Ponader KC (2004) Two common North American Diatoms, Achnanthidium rivulare sp. nov. and a. deflexum (Reimer) kingston: Morphology, ecology and comparison with related species. Diatom Research 19(1): 33-57

36. John J (2018) The diatom flora of Australia. Diatoms from Tasmania: Taxonomy and biogeography. Koeltz Scientific Books 2: 1-656.

37. Danielidis DB, Mann DG (2003) New species and new combinations in the genus Seminavis (Bacillariophyta). Diatom Research 18: 21-39.

38. Khairy HM, Shaltout KH, El-Sheekh MM, Eassa DI (2017) A checklist of diatom species reported from the Egyptian mediterranean lakes. Annual Research and Review in Biology 19: 1-29.

39. Gandhi HP (1999) Fresh water diatoms of central Gujarat with a review and some others. Bishen Singh Mahendra Pal Singh.

40. Clavero-Oms E (2004) Diatomees dambients hipersalins costaners Taxonomia, distribució i emprentes en el registre sedimentari. Universitat de Barcelona, Barcelona.

41. Wachnicka AH, Gaiser EE (2007) Characterization of A. Seminavis from South Florida, U.S.A. Diatom Research 22(2): 387-455.

42. López-Fuerte FO, Siqueiros-Beltrones DA (2016) A checklist of marine benthic diatoms (Bacillariophyta) from Mexico. Phytotaxa 283(3): 201-258.

43. N Guessan KR, Aboua BRD, Tison-Rosebery J, Ouattara A, Kouamelan EP (2018) Biodiversity and ecology of epilithic diatoms in the Agnéby River, Ivory Coast. African Journal of Aquatic Science 43(2): 131-140.

44. Medlin LK, Kaczmarska I (2004) Evolution of the diatoms: V. Morphological and cytological support for the major clades and a taxonomic revision. Phycologia 43(3): 245-270.

45. Medlin LK (2016) Evolution of the diatoms: Major steps in their evolution and a review of the supporting molecular and morphological evidence. Phycologia 55(1): 79-103.

46. Guiry MD, Guiry GM (2019) AlgaeBase. World-wide electronic publication. National University of Ireland, Galway. 
This work is licensed under Creative Commons Attribution 4.0 Licens

DOI: 10.19080/OFOAJ.2021.13.555874
Your next submission with Juniper Publishers will reach you the below assets

- Quality Editorial service

- Swift Peer Review

- Reprints availability

- E-prints Service

- Manuscript Podcast for convenient understanding

- Global attainment for your research

- Manuscript accessibility in different formats ( Pdf, E-pub, Full Text, Audio)

- Unceasing customer service

Track the below URL for one-step submission https://juniperpublishers.com/online-submission.php 